\title{
INVOLUTIONS ON HOMOTOPY SPHERES AND THEIR GLUING DIFFEOMORPHISMS
}

\author{
BY

\section{CHAO-CHU LIANG}

ABSTRACT. Let $h S\left(P^{2 n+1}\right)$ denote the set of equivalence classes of smooth fixed-point free involutions on $(2 n+1)$-dimensional homotopy spheres. Browder and Livesay defined an invariant $\sigma\left(\Sigma^{2 n+1}, T\right)$ for each $\left(\Sigma^{2 n+1}, T\right) \in$ $h S\left(P^{2 n+1}\right)$, where $\sigma \in Z$ if $n$ is odd, $\sigma \in Z_{2}$ if $n$ is even. They showed that for $n \geqslant 3, \sigma\left(\Sigma^{2 n+1}, T\right)=0$ if and only if $\left(\Sigma^{2 n+1}, T\right)$ admits a codim 1 invariant sphere. For any $\left(\Sigma^{2 n+1}, T\right)$, there exists an $A$ equivariant diffeomorphism $f$ of $S^{n} \times S^{n}$ such that $\left(\Sigma^{2 n+1}, T\right)=\left(S^{n} \times D^{n+1}, A\right) \cup\left(D^{n+1} \times S^{n}, A\right)$, where $A$ denotes the antipodal map. Let $\beta(f)=\sigma\left(\Sigma^{2 n+1}, T\right)$. In the case $n$ is odd, we can show that the Browder-Livesay invariant is additive: " $\beta(f g)=\beta(f)+$ $\beta(g)$ ". But if $n$ is even, then there exists $f$ and $g$ such that $\beta(g f)=\beta(g)+\beta(f) \neq$ $\beta(f g)$. Let $D_{0}\left(S^{n} \times S^{n}, A\right)$ be the group of concordance classes of $A$ equivariant diffeomorphisms which are homotopic to the identity map of $S^{n} \times s^{n}$. We can prove that "For $n \equiv 0,1,2 \bmod 4, h S\left(P^{2 n+1}\right)$ is in 1-1 correspondence with a subgroup of $D_{0}\left(S^{n} \times S^{n}, A\right)$ ". As an application of these theorems, we demonstrated that "Let $\Sigma_{0}^{8 k+3}$ denote the generator of $b P_{8 k+4}$. Then the number of $\left(\Sigma_{0}^{8 k+3}, T\right)$ 's with $\sigma\left(\Sigma_{0}^{8 k+3}, T\right)=0$ is either 0 or equal to the number of $\left(S^{8 k+3}, T\right)$ 's with $\sigma\left(S^{8 k+3}, T\right)=0$, where $S^{8 k+3}$ denotes the standard sphere".

0. Introduction. In [7], [8], Browder and Livesay studied differentiable fixed-point free involutions on homotopy spheres. They defined the BrowderLivesay desuspension invariant $\sigma$ for each free involution $\left(\Sigma^{m}, T\right): \sigma\left(\Sigma^{m}, T\right)=0$, for $m$ even; $\sigma\left(\Sigma^{m}, T\right) \in Z$, for $m=4 k+3$; $\sigma\left(\Sigma^{m}, T\right) \in Z_{2}$, for $m=4 k+1$. For $m \geqslant 6$, they proved that $\sigma\left(\Sigma^{m}, T\right)=0$ if and only if $\left(\Sigma^{m}, T\right)$ admits a codim 1 invariant subsphere $\left(S^{m-1}, T \mid S^{m-1}\right)$ embedded in it. It was shown by several people that all these desuspension invariants can be realized, [2], [6], [21], [33], and [34] etc.

Livesay and Thomas, [20], showed that any $\left(\Sigma^{2 n+1}, T\right)$ can be obtained by gluing $\left(S^{n} \times D^{n+1}, A\right)$ and $\left(D^{n+1} \times S^{n}, A\right)$ together by an $A$-equivariant diffeomorphism $f$ of their boundaries, where $A$ is the antipodal map. We shall denote this $\left(\Sigma^{2 n+1}, T\right)$ by $\left(\Sigma_{f}, T_{f}\right)$. The purpose of this paper is to investigate the rela-

Received by the editors August 7, 1974.

AMS (MOS) subject classifications (1970). Primary 57E25, 57E30, 57D65, 55E05.

Key wrods and phrases. Free differentiable involutions, Browder-Livesay invariant, equivariant diffeomorphism, concordance group of diffeomorphisms, curious involutions. 
tion between the free involutions on odd dimensional homotopy spheres and their gluing diffeomorphisms.

Let $A$ denote the antipodal map on $S^{n} \times S^{n}$, defined by $A(x, y)=(-x,-y)$ for $(x, y) \in S^{n} \times S^{n}$. An $A$-equivariant map $f: S^{n} \times S^{n} \rightarrow S^{n} \times S^{n}$ is a map such that $f A=A f$, we shall call $f$ an $A$-map. An $A$-map $f$ induces a map $f^{\prime}$ : $S^{n} \times S^{n} / A \rightarrow S^{n} \times S^{n} / A$, where $S^{n} \times S^{n} / A$ denotes the orbit space of $S^{n} \times S^{n}$ under the action of $A$. If $f^{\prime}$ is a diffeomorphism (or a homotopy equivalence), we will call $f$ an $A$-diffeomorphism (or an $A$-homotopy equivalence). Considering the action of $A \times$ identity on $S^{n} \times S^{n} \times[0,1]$, we have the notion of $A$-homotopy and $A$-concordance etc. (see $\$ 2$ below).

Let $D\left(S^{n} \times S^{n}, A\right)$ denote the group of $A$-concordance classes of $A$-diffeomorphisms of $S^{n} \times S^{n}$. Define $J_{2 n+1}=\left\{f \in D\left(S^{n} \times S^{n}, A\right) \mid f\right.$ is homotopic to identity\}, (but $f$ might not be $A$-homotopic to identity). $J_{2 n+1}$ is a subgroup of $D\left(S^{n} \times S^{n}, A\right)$. We will show that the gluing diffeomorphism can always be chosen from $J_{2 n+1}$, (§4).

Two involutions $(\Sigma, T)$ and $\left(\Sigma^{\prime}, T^{\prime}\right)$ are called equivalent, $(\Sigma, T)=\left(\Sigma^{\prime}, T^{\prime}\right)$, if there exists an orientation-preserving diffeomorphism $f: \Sigma \rightarrow \Sigma^{\prime}$ such that $f \circ T$ $=T^{\prime} \circ f$. Let $h S\left(P^{2 n+1}\right)$ denote the set of homotopy smoothings of $P^{2 n+1}$, [34], which is also the set of equivalence classes of differentiable free involutions on $(2 n+1)$-homotopy spheres, [21]. In $\S 5$ below, we will prove that for $n \equiv 0,1,2$ $\bmod 4, h S\left(P^{2 n+1}\right)$ is in 1-1 correspondence with a subgroup $G_{2 n+1}$ of $J_{2 n+1}$. Thus, in these cases, $h S\left(P^{2 n+1}\right)$ forms a group by carrying over the composition law of diffeomorphisms in $G_{2 n+1}$.

Also, we will show in Theorem 6.15 below that the Browder-Livesay index invariant is additive. For $m=4 k+3, f$ and $g \in J_{m}$, we have $\sigma\left(\Sigma_{f}, T_{f}\right)+$ $\sigma\left(\Sigma_{g}, T_{g}\right)=\sigma\left(\Sigma_{f g}, T_{f g}\right)$.

From Theorems 5.2 and 6.15, we can deduce the following theorem concerning the curious involutions in the sense of [13]. Let $\Sigma_{0}^{n}$ denote the generator of $b P_{n+1},[17] . S^{n}$ denotes the standard sphere.

THEOREM 8.2. For $n=8 k+3$, the number of curious involutions $\left(\Sigma_{0}^{n}, T\right)$ with $\sigma\left(\Sigma_{0}^{n}, T\right)=0$ is either 0 or equal to the number of involutions $\left(S^{n}, T\right)$ with $\sigma\left(S^{n}, T\right)=0$.

Everything considered here is assumed to be in the smooth category.

This paper is essentially the author's doctoral thesis written at Cornell University. I wish to thank my thesis advisor, Professor G. R. Livesay, for his constant help and encouragement. I also wish to thank Professors I. Berstein, P. J. Kahn, and H. C. Wang for many useful discussions.

1. Livesay-Thomas decomposition theorem. We have the following theorem from [20]. 
THEOREM 1.1. For any free involution on a homotopy sphere $\left(\Sigma^{2 n+1}, T\right)$, $n \geqslant 3$, there exists an A-diffeomorphism $g$ of $S^{n} \times S^{n}$ such that $\left(\Sigma^{2 n+1}, T\right)=$ $\left(S^{n} \times D^{n+1}, A\right) \cup_{g}\left(D^{n+1} \times S^{n}, A\right)$, denoted by $\left(\Sigma_{g}, T_{g}\right)$.

Note. $M \cup_{g} N$ denotes a manifold obtained by gluing two manifolds $M$ and $N$ together by a diffeomorphism $g: M_{0} \rightarrow N_{0}$, where $M_{0} \subseteq \partial M$ and $N_{0} \subseteq \partial N$.

We shall prove the following proposition in $\$ 4$ below.

Proposition 1.2. For any $\left(\Sigma^{2 n+1}, T\right)$, where $n \neq 3,7$, there exists an $A$ diffeomorphism $g$ of $S^{n} \times S^{n}$ such that $g$ is homotopic to the identity and $\left(\Sigma^{2 n+1}, T\right)=\left(\Sigma_{g}, T_{g}\right)$.

Notation. Let " " denote homotopic, and " $A$ " denote $A$-homotopic.

LEMMA 1.3. If $g$ is an A-diffeomorphism of $S^{n} \times S^{n}$ such that $g \sim$ Id (the identity), then there exists a pair of A-homotopy equivalences $f_{1}, f_{2}$ of $S^{n} \times S^{n}$ such that $f_{1} \sim f_{2} \sim \mathrm{Id}$, where $f_{1}\left(f_{2}\right)$ extends to an A-homotopy equivalence $h_{1}\left(h_{2}\right)$ of $S^{n} \times D^{n+1}\left(D^{n+1} \times S^{n}\right)$; and $g \sim A f_{2} f_{1}$.

Proof. Let $p_{j}$ denote the projection of $S^{n} \times S^{n}$ onto the $j$ th factor $S_{j}^{n}$, $j=1$ or $2 . p_{j}$ is $A$-equivariant: $p_{j}(A x, A y)=A\left(p_{j}(x, y)\right)$. Let $g_{j}=p_{j} \circ g$. We define $f_{1}$ by $f_{1}(x, y)=\left(x, g_{2}(x, y)\right) \cdot g^{-1}$ is also an $A$-diffeomorphism. Let $k_{j}=$ $p_{j} \circ g^{-1}$. We define $f_{3}$ by $f_{3}(x, y)=\left(k_{1}(x, y), y\right)$.

$$
\begin{aligned}
f_{3} \circ g(x, y) & =f_{3}\left(g_{1}(x, y), g_{2}(x, y)\right)=\left(k_{1}\left(g_{1}(x, y), g_{2}(x, y)\right), g_{2}(x, y)\right) \\
& =\left(x, g_{2}(x, y)\right)=f_{1}(x, y) .
\end{aligned}
$$

$f_{1}$ and $f_{3}$ are obviously $A$-maps.

Since $g \sim \mathrm{Id},\left(x_{0}, y\right) \rightarrow\left(x_{0}, g_{2}\left(x_{0}, y\right)\right)$ is a degree 1 map of $x_{0} \times S^{n}$ to itself for each $x_{0} \in S^{n}$. Hence $f_{1} \mid x_{0} \times S^{n} \rightarrow x_{0} \times S^{n}$ is a homotopy equivalence for each $x_{0} \in S^{n}$. We have a locally trivial fibre bundle $S_{2}^{n} \rightarrow S^{n} \times S^{n} / A$ $\rightarrow S_{1}^{n} / A$, with base space $S_{1}^{n} / A$ and fibre $S_{2}^{n}$. The map $f_{1}^{\prime}: S^{n} \times S^{n} / A \rightarrow S^{n} \times$ $S^{n} / A$, induced by $f_{1}$, is fibre preserving. The restriction of $f_{1}^{\prime}$ to each fibre is a homotopy equivalence. Hence $f_{1}^{\prime}$ is a fibre homotopy equivalence by a theorem of Dold [10], and so $f_{1}$ is an $A$-homotopy equivalence. Similarly, we can show that $f_{3}$ induces a fibre homotopy equivalence $f_{3}^{\prime}$ of the bundle $S_{1}^{n} \rightarrow S^{n} \times S^{n} / A$ $\rightarrow S_{2}^{n} / A$. Let $f_{2}^{\prime}$ be the fibre homotopy inverse of $f_{3}^{\prime}$, and write $f_{2}$ for the double cover of $f_{2}^{\prime}$ such that $f_{2}$ is the $A$-homotopy inverse for $f_{3}$. Now since $f_{3} \circ g=f_{1}$, it follows that $g \sim A f_{2} f_{1}$.

Since the $A$-map $g_{2}: S^{n} \times S^{n} \rightarrow S_{2}^{n}$ extends to an $A$-map $\bar{g}_{2}: S^{n} \times D^{n+1}$ $\rightarrow D^{n+1}$ by radial extension, we define an $A$-homotopy equivalence $h_{1}$ of $S^{n} \times$ $D^{n+1}$ by $h_{1}(x, y)=\left(x, \bar{g}_{2}(x, y)\right)$. An $A$-homotopy equivalence $h_{2}$ of $D^{n+1} \times$ $S^{n}$ can be defined similarly. $f_{1} \sim f_{2} \sim$ Id follows from [19, 2.5]. Q.E.D. 
Lemma 1.4. Suppose $\left(\Sigma^{2 n+1}, T\right)=\left(\Sigma_{g}, T_{g}\right)=S^{n} \times D^{n+1} \cup_{\mathrm{Id}} S^{n} \times S^{n}$ $\times I \cup_{g} D^{n+1} \times S^{n}$ for an A-diffeomorphism $g$ as in (1.2). Then there exists an equivariant homotopy equivalence $F:\left(\Sigma^{2 n+1}, T\right) \rightarrow\left(S^{2 n+1}, A\right)=S^{n} \times D^{n+1}$ $\cup_{\text {Id }} S^{n} \times S^{n} \times I \cup_{\text {Id }} D^{n+1} \times S^{n}$ such that each summand is mapped into the corresponding one by an A-homotopy equivalence.

Proof. For such an $A$-diffeomorphism $g$, there exists $f_{1}, f_{2}, h_{1}, h_{2}$ as in (1.3). Write $f_{2}^{-1}, h_{2}^{-1}$ for $A$-homotopy inverses of $f_{2}, h_{2}$. Let $H$ be an $A$-homotopy between $f_{2}^{-1} \circ g$ and $f_{1}$ with $H(x, 0)=f_{1}(x)$. We then define $F=h, H$, $h_{2}^{-1}$ on each summand as follows:

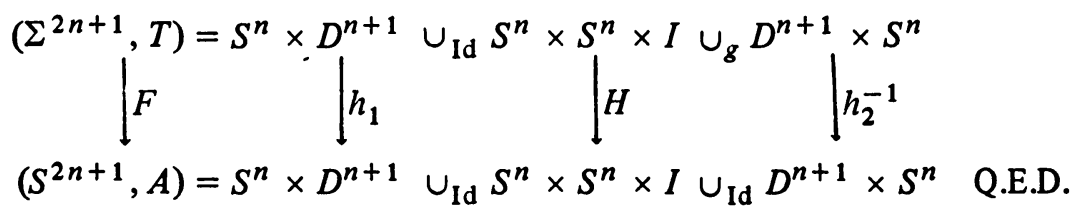

An invariant $m$-manifold for $\left(\Sigma^{k}, T\right)$ is an embedded $m$-manifold $M^{m} \subseteq \Sigma^{k}$ which is invariant under $T$. An invariant $M^{m}$ for $\left(\Sigma^{k}, T\right)$ is called characteristic if there is an equivariant map $F:\left(\Sigma^{k}, T\right) \rightarrow\left(S^{N}, A\right), N \geqslant k$, such that $F$ is transverse to $S^{N+m-k} \subseteq S^{N}$ and $F^{-1}\left(S^{N+m-k}\right)=M^{m}$.

Proposition 1.5. Let $\left(\Sigma^{2 n+1}, T\right)=\left(\Sigma_{g}, T_{g}\right)$ for an A-diffeomorphism $g$ as in (1.2). If one of $f_{1}, f_{2}$ corresponding to $g$ in (1.3) is A-homotopic to Id, then $\left(\Sigma^{2 n+1}, T\right)$ admits $S^{m}$, where $m=1, \ldots, n$, as characteristic spheres, such that $\left(S^{m}, T \mid S^{m}\right)$ is conjugate to $\left(S^{m}, A\right)$.

Proof. Suppose $f_{1} \sim A$ Id. We take $H$ in (1.4) to be an $A$-homotopy between $f_{2}^{-1} \circ g$ and Id, and $h_{1}=$ Id. Let $S^{m}=S^{m} \times 0 \subseteq S^{n} \times D^{n+1}$. From (1.4), we see that $F^{-1}\left(S^{m}\right)=h_{1}^{-1}\left(S^{m}\right)=S^{m}$. Since $h_{1}=\mathrm{Id},\left(S^{m}, T \mid S^{m}\right)$ is equivalent to $\left(S^{m}, A\right)$. Q.E.D.

REMARK 1.6. In [6], Browder showed that there exists a smooth involution $\left(\Sigma_{0}^{4 k+1}, T_{0}\right)$ which admits no $m$-dimensional homotopy sphere, $m \neq 4 l+1$, as characteristic manifold. Hence, any $A$-diffeomorphism $g$ of $S^{n} \times S^{n}$ such that $\left(\Sigma_{g}, T_{g}\right)=\left(\Sigma_{0}^{4 k+1}, T_{0}\right)$ is not $A$-homotopic to the identity by (1.5).

2. Nonuniqueness of the decomposition. The decomposition for $\left(\Sigma^{2 n+1}, T\right)$ in (1.1) is not unique: we may have different $A$-diffeomorphisms $f$ and $g$ such that $\left(\Sigma^{2 n+1}, T\right)=\left(\Sigma_{f}, T_{f}\right)=\left(\Sigma_{g}, T_{g}\right)$. But we have the following

Proposition 2.1 [20]. For $n \geqslant 3,\left(\Sigma^{2 n+1}, T\right)=\left(\Sigma_{f}, T_{f}\right)=\left(\Sigma_{g}, T_{g}\right)$ iff there exist Addffeomorphisms $H: S^{n} \times D^{n+1} \rightarrow S^{n} \times D^{n+1}$ and $K: D^{n+1} \times S^{n}$ $\rightarrow D^{n+1} \times S^{n}$ such that, when we restrict our attention to the boundary, $g=K f H$.

Two diffeomorphisms $f$ and $g$ of a manifold $M$ are called concordant, if there exists a diffeomorphism $H: M \times[0,1] \rightarrow M \times[0,1]$ such that $H(x, 0)=$ 
$(f(x), 0), H(x, 1)=(g(x), 1)$. Similarly, we have the notion of $A$-concordance between two $A$-diffeomorphisms. If $f$ and $g$ are $A$-concordant diffeomorphisms of $S^{n} \times S^{n}$, then $\left(\Sigma_{f}, T_{f}\right)=\left(\Sigma_{g}, T_{g}\right)$, which can be seen by constructing an equivariant diffeomorphism $F$ between them as follows:

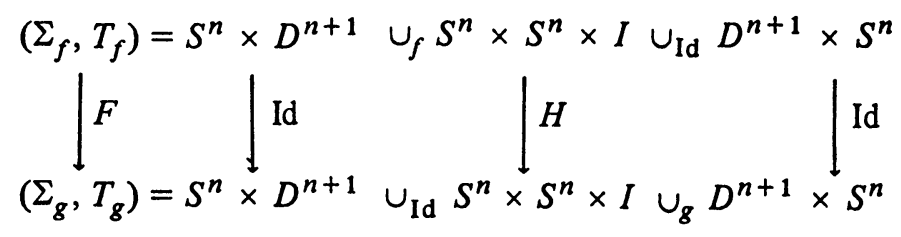

where $H^{-1}$ is an $A$-concordance between $f$ and $g$. Q.E.D.

Now, we are going to determine the $A$-diffeomorphisms $H$ and $K$ in (2.1) within $A$-concordance classes.

Definition. A bundle map $f_{a}$ for $S^{n} \times S^{n}$ over the first factor is a map of the form $f_{a}(x, y)=(x, a(x) \cdot y)$, where the homotopy class $\{a\} \in \pi_{n}\left(S O_{n+1}\right)$ and $a$ is a smooth map of $S^{n}$ to $S O_{n+1}$.

A bundle map $f_{a}$ is a diffeomorphism. If $\{a\},\{b\} \in \pi_{n}\left(S O_{n+1}\right)$ are homotopic, then $f_{a}$ and $f_{b}$ are concordant. Conversely, if $f_{a}$ and $f_{b}$ are concordant, then it was shown in $[19,5.2]$ that $a$ and $b$ are homotopic. Actually, we have

LEMmA 2.2. [19]. The concordance classes of orientation-preserving diffeomorphisms of $S^{n} \times S^{n}$, which can be extended to orientation-preserving diffeomorphisms of $S^{n} \times D^{n+1}$, are in 1-1 correspondence with $\pi_{n}\left(S O_{n+1}\right)$.

We write $S^{n}=\left\{x=\left(x_{0}, \ldots, x_{n}\right) \in R^{n+1} \mid \Sigma_{i=0}^{n} x_{i}^{2}=1\right\} . A$ acts on $S^{n}$ as an $(n+1)$-square matrix with -1 on its diagonal and 0 elsewhere. For $(x, y)$ $\in S^{n} \times S^{n}, A(x, y)=(-x,-y)$.

Lemma 2.3. Let $b \in \pi_{n}\left(S O_{n+1}\right)$. Then the bundle map $f_{b}$ defined above is an A-equivariant bundle map if and only if $b$ factors through $P^{n}$ by the double covering map $\pi: S^{n} \rightarrow P^{n}$.

PRoof. $f_{b} A=A f_{b} \Longleftrightarrow f_{b} A(x, y)=A f_{b}(x, y) \Longleftrightarrow(-x, b(-x) \cdot(-y))=$ $(-x,-b(x) \cdot y) \Longleftrightarrow b(-x) \cdot A \cdot y=A \cdot b(x) \cdot y$. Since $A$ lies in the center of $S O_{n+1}$, we have $b(x) \cdot y=b(-x) \cdot y$ for all $y \in S^{n}$. Hence $f_{b} A=A f_{b}$ iff $b(x)$ $=b(-x)$ for all $x \in S^{n}$ iff $b$ factors through $\pi: S^{n} \rightarrow P^{n}$. Q.E.D.

LEMma 2.4. Every A-diffeomorphism of $S^{n} \times S^{n}, n \geqslant 3$, which can be extended to an orientation-preserving A-diffeomorphism of $S^{n} \times D^{n+1}$ is A-concordant to an A-equivariant bundle map over the first factor.

Proof. Let $f$ be such an $A$-diffeomorphism, and $h$ be its $A$-equivariant extension to $S^{n} \times D^{n+1} . f^{\prime}\left(h^{\prime}\right)$ denotes the map induced by $f(h)$ on the orbit space $S^{n} \times S^{n} / A\left(S^{n} \times D^{n+1} / A\right)$. Let $i^{\prime}: S^{n} \times 0 / A \rightarrow S^{n} \times D^{n+1} / A$ be the 
inclusion. $h^{\prime} \mid S^{n} \times 0 / A$ and $i^{\prime}$ are homotopic by [24]. Hence, they are isotopic by a theorem of Haefliger, [12]. By the equivariant isotopy extension theorem, [28], there exists an $A$-equivariant diffeomorphism $H$ of $S^{n} \times D^{n+1}$ such that $H$ is equivariantly isotopic to identity, $H \mid S^{n} \times S^{n}=$ identity, and $H \circ h \mid S^{n} \times 0 / A=$ $i$, the inclusion. Let $B^{n+1}$ be a small disk in $D^{n+1}$, with $B^{n+1}$ and $D^{n+1}$ concentric. Both $S^{n} \times B^{n+1}$ and $H \circ h\left(S^{n} \times B^{n+1}\right)$ are equivariant tubular neighborhoods of $S^{n} \times 0$ in $S^{n} \times D^{n+1}$. Then by the uniqueness of the equivariant tubular neighborhoods, [4, p. 310], there exists an $A$ equivariant diffeomorphism $G$ of $S^{n} \times D^{n+1}$ such that $G$ is $A$-equivariantly isotopic to identity, $G \mid S^{n} \times S^{n}$ $=\mathrm{Id}$, and $G \circ H \circ h \mid S^{n} \times B^{n+1}$ is an $A$-equivariant bundle map covering the identity on $S^{n}$. The restriction of $G \circ H \circ h$ to $S^{n} \times D^{n+1}$-interior $S^{n} \times B^{n+1}$ gives us an $A$-concordance between $f$ and an $A$-equivariant bundle map. Q.E.D.

Similarly, every $A$-diffeomorphism of $S^{n} \times S^{n}$ which extends equivariantly to $D^{n+1} \times S^{n}$ is $A$-concordant to an $A$-bundle over the second factor.

Proposition 2.5. The A-concordance classes of orientation-preserving $A$ diffeomorphism $H($ or $K)$ in (2.1) are in 1-1 correspondence with the Image $\pi^{*}$ of $\left[P^{n}, S O_{n+1}\right]$ in $\left[S^{n}, S O_{n+1}\right]$.

Proof. Let $h$ be an $A$-diffeomorphism of $S^{n} \times S^{n}$, which can be extended equivariantly to $S^{n} \times D^{n+1}$. $h$ is $A$-concordant to an $A$-equivariant bundle map $f_{b}$ by (2.4), where $b \in \pi_{n}\left(S O_{n+1}\right)$. From (2.3), we know that $b$ factors through $\pi: S^{n} \rightarrow P^{n}$, i.e. $b \in$ Image $\pi^{*}$.

The above correspondence $H \rightarrow b$ is well-defined. If $H^{\prime}$ is $A$-concordant to $H$, and $b^{\prime} \in$ Image $\pi^{*}$ corresponds to $H^{\prime}$, then $f_{b}$ and $f_{b^{\prime}}$ are $A$-concordant, hence concordant. $b$ is homotopic to $b^{\prime}$ by (2.2). This correspondence is $1-1$ and onto, since the mapping given by $b \rightarrow f_{b}$ for $b \in$ Image $\pi^{*}$ is its inverse. Q.E.D.

3. The image of $\pi^{*}:\left[P^{n}, S O_{n+1}\right] \rightarrow\left[S^{n}, S O_{n+1}\right]$. In this section, we will compute $\left[P^{n}, S O_{n+1}\right]$ and its image under $\pi^{*}:\left[P^{n}, S O_{n+1}\right] \rightarrow\left[S^{n}, S O_{n+1}\right]$. Let us first recall some facts about $\pi_{n}\left(\mathrm{SO}_{n+1}\right)$, which, for instance, can be found in [16] or [18].

Let $s_{m *}: \pi_{k}\left(\mathrm{SO}_{m}\right) \rightarrow \pi_{k}\left(\mathrm{SO}_{m+1}\right)$ denote the homomorphism induced by the natural embedding $s: \mathrm{SO}_{n} \rightarrow \mathrm{SO}_{n+1}$. Consider the following exact sequence,

$$
\ldots \stackrel{\partial_{m}}{\longrightarrow} \pi_{k}\left(S O_{m}\right) \stackrel{s_{m *}}{\longrightarrow} \pi_{k}\left(S O_{m+1}\right) \stackrel{q_{m *}}{\longrightarrow} \pi_{k}\left(S^{m}\right) \stackrel{\partial_{m}}{\longrightarrow} \pi_{k-1}\left(S O_{m}\right) \rightarrow \cdots .
$$

Let $l_{m}$ denote the generator of $\pi_{m}\left(S^{m}\right)$. Write $\tau_{m}=\partial_{m+1} l_{m+1} \in \pi_{m}\left(S O_{m+1}\right)$. Putting $m=n, n+1$ in the above exact sequence, we have the following proposition from [18]:

Proposition 3.1. For $n$ odd $, \neq 1,3,7, \pi_{n}\left(\mathrm{SO}_{n+1}\right)$ is the direct sum of two cyclic subgroups image $\partial_{n+1}$ and image $s_{n *}$. Moreover, $s_{n+1 *}$ : image $s_{n *} \subseteq$ 
$\pi_{n}\left(S O_{n+1}\right) \rightarrow \pi_{n}\left(S O_{n+2}\right)$ is an isomorphism. For $n$ even, $\pi_{n}\left(S O_{n+1}\right)$ is the direct sum of image $\partial_{m+1}$ and a certain cyclic subgroup $G$ such that $s_{n+1 *}: G \subseteq$ $\pi_{n}\left(\mathrm{SO}_{n+1}\right) \rightarrow \pi_{n}\left(\mathrm{SO}_{n+2}\right)$ is an isomorphism.

From now on, we will write $\tau$ for $\tau_{n}=\partial_{n+1} \iota_{n+1}$, if no confusion will arise. Let $\sigma$ denote the generator of the other cyclic summand of $\pi_{n}\left(S O_{n+1}\right)$. Here we list the values of $\pi_{n}\left(S O_{n+1}\right)$ and $\pi_{n}\left(S O_{n+2}\right)=\pi_{n}(S O)$ for $n>3$ and $n \neq 7$, from [16].

$\begin{array}{ccccccccc}n(\bmod 8) & 0 & 1 & 2 & 3 & 4 & 5 & 6 & 7 \\ \pi_{n}\left(S O_{n+1}\right) & Z_{2}+Z_{2} & Z+Z_{2} & Z_{2} & Z+Z & Z_{2} & Z & Z_{2} & Z+Z \\ & \tau, \sigma & \tau, \sigma & \tau & \tau, \sigma & \tau & \tau & \tau & \tau, \sigma \\ \pi_{n}(S O) & Z_{2} & Z_{2} & 0 & Z & 0 & 0 & 0 & Z\end{array}$

Consider the maps $\pi: S^{n} \rightarrow P^{n}, i: P^{n} \rightarrow P^{n+1}, j: P^{n} \rightarrow S^{n}$; which are the double covering, the inclusion, and the map pinching the complement of an open ball to a point. $j$ generates $\left[P^{n}, S^{n}\right]=H^{n}\left(P^{n}\right)=Z$ or $Z_{2}, n$ odd or even; by Hopf's theorem, [23].

The element $\tau \in \pi_{n}\left(S O_{n+1}\right)$ is also the characteristic map for the tangent bundle of $S^{n+1}$, [14] or [30]. We can choose a representative for $\tau$ such that $\tau(x)=\tau(-x)$ for $x \in S^{n},[14]$, which is defined as follows: let $\alpha: S^{n} \rightarrow S O_{n+1}$ be the map defined by the requirement that $\alpha(x)$ be a reflection through the hyperplane in $R^{n+1}$ orthogonal to $x$, and let $e$ denote the north pole of $S^{n}$, then we have $\tau(x)=\alpha(x) \alpha(e),\left[14\right.$, p. 89]. Hence this $\tau$ factors through $\pi: S^{n} \rightarrow P^{n}$, and $\tau_{p} \in\left[P^{n}, S O_{n+1}\right]$ is defined by $\tau=\tau_{p} \pi$.

Let $q: S O_{n+1} \rightarrow S^{n}$ denote the projection in the fibration $S O_{n} \rightarrow S O_{n+1}$ $\rightarrow S^{n}$.

Proposition 3.2. The following diagram is homotopically commutative.

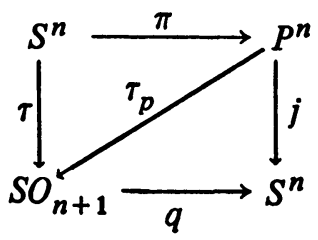

Proof. We have to show that $g \tau_{p}$ is homotopic to $j$. From the above description of $\tau, \tau(x)=\alpha(x) \alpha(e)$; we see that $q \tau(x)=\alpha(x) \alpha(e)=\alpha(x)(-e)$, which is the point on $S^{n}$ obtained by moving $e$ toward $x$ along the great circle passing through $e$ and $x$ by an angle twice the angle between $e$ and $x$. We note that $q \tau$ maps the interior of the northern hemisphere $D_{+}^{n}$ of $S^{n}$ onto $S^{n}-\{e\}$ as a homeomorphism, and maps the equator $S^{n-1}$ to $-e$. Since $\pi$ maps interior $D_{+}^{n}$ homeomorphically onto $P^{n}-P^{n-1}$, and $q \tau=q \tau_{p} \pi$, we see that $q \tau_{p}$ is just the 
map pinching the complement of an open ball to a point, which is $j$. Q.E.D.

LEMMA 3.3. $\tau j$ is null-homotopic in $\left[P^{2 k}, \mathrm{SO}_{2 k+1}\right]$.

ProOF. We have $j j=\tau_{p} \pi j: P^{2 k} \rightarrow S^{2 k} \rightarrow S O_{2 k+1}$. Consider $f=\pi j:$ $P^{2 k} \rightarrow P^{2 k}$. Since $f$ factors through $S^{2 k}, f_{*}\left(\pi_{1}\left(P^{2 k}\right)\right)=0$. Hence $f$ is a nonorientation-true map in the language of [25]. By Theorem 1.2 of [25], (see also $[25,1.3(d)]), f$ is null-homotopic. Q.E.D.

Let $K O^{-k}(-)$ denote the reduced real $K$-theory. We have $K O^{-k}(X)=$ $\left[\Sigma^{k} X, B_{S O}\right]$ for any finite $C W$ complex $X,[14] . K O^{-1}\left(P^{n}\right)=\left[\Sigma P^{n}, B_{S O}\right]=$ $\left[P^{n}, \Omega B_{S O}\right]=\left[P^{n}, S O\right]$, the latter one is equal to $\left[P^{n}, S O_{n+2}\right]$ because $(S O$, $\left.S O_{n+2}\right)$ is $(n+1)$-connected.

For any fibration $F \rightarrow E \rightarrow B$, and any finite $C W$ complex $X$, there is a fibre mapping sequence $\cdots \rightarrow[X, \Omega E] \rightarrow[X, \Omega B] \rightarrow[X, F] \rightarrow[X, E] \rightarrow$ $[X, B],[23]$, which is exact.

Lemma 3.4. For $n$ odd, $Z \rightarrow\left[P^{n}, S O_{n+1}\right] \rightarrow\left[P^{n}, S O_{n+2}\right] \rightarrow 0$ is exact.

For $n$ even, $Z_{2} \rightarrow\left[P^{n}, S O_{n+1}\right] \rightarrow\left[P^{n}, S O_{n+2}\right] \rightarrow 0$ is exact.

Proof. Substitute $S O_{n+1} \rightarrow S O_{n+2} \rightarrow S^{n+1}$ and $P^{n}$ into the above fibre mapping sequence. $\left[P^{n}, S^{n+1}\right]=0$. $\left[P^{n}, \Omega S^{n+1}\right]=\left[\Sigma P^{n}, S^{n+1}\right]=$ $H^{n+1}\left(\Sigma P^{n}\right)=H^{n}\left(P^{n}\right)=Z, n$ odd; $=Z_{2}, n$ even. Q.E.D.

We also need the following from [6] or [11].

Proposition 3.5. $K O^{-1}\left(P^{m}\right)=Z_{2}$, for $m \not \equiv 3 \bmod 4 ; K O^{-1}\left(P^{m}\right)=Z+$ $Z_{2}$, for $m \equiv 3 \bmod 4$, where the $Z$ summand is the image of $K O^{-1}\left(S^{m}\right)$ under the degree 1 map $j: P^{m} \rightarrow S^{m}$. The inclusion $P^{k} \subseteq P^{m}$ induces $K O^{-1}\left(P^{m}\right) \rightarrow$ $K O^{-1}\left(P^{k}\right)$, which is an isomorphism on $Z_{2}$ and annihilates the $Z$ factor.

Replacing $P^{k}$ by the mapping cylinder $M_{\pi}$ of $\pi: S^{k} \rightarrow P^{k}$, we can change $\pi$ into a cofibration $\pi^{\prime}: S^{k} \rightarrow M_{\pi}$, and we may consider $i: P^{k} \rightarrow P^{k+1}$ as the cofibre. For a simple space $X$, we have the following Puppe exact sequence, [27],

$$
\begin{gathered}
\cdots \longrightarrow\left[\Sigma P^{k}, X\right] \stackrel{\Sigma \pi^{*}}{\longrightarrow} \pi_{k+1}(X) \stackrel{j^{*}}{\longrightarrow}\left[P^{k+1}, X\right] \\
\stackrel{i^{*}}{\longrightarrow}\left[P^{k}, X\right] \stackrel{\pi^{*}}{\longrightarrow} \pi_{k}(X) .
\end{gathered}
$$

Putting $k=n-1$ and $X=S O_{n+1}$ in the above Puppe sequence, we have the following exact sequence:

$$
\begin{aligned}
\cdots & \longrightarrow \pi_{n}\left(S O_{n+1}\right) \stackrel{j^{*}}{\longrightarrow}\left[P^{n}, S O_{n+1}\right] \\
& \stackrel{i^{*}}{\longrightarrow}\left[P^{n-1}, S O_{n+1}\right] \stackrel{\pi^{*}}{\longrightarrow} \pi_{n-1}\left(S O_{n+1}\right) .
\end{aligned}
$$

For $n$ odd, $j \pi: S^{n} \rightarrow P^{n} \rightarrow S^{n}$ is of degree 2. Hence $\pi^{*} j^{*}: \pi_{n}\left(S O_{n+1}\right) \rightarrow$ $\left[P^{n}, S O_{n+1}\right] \rightarrow \pi_{n}\left(S O_{n+1}\right)$ is just the multiplication by 2 . 
If $n \equiv 3 \bmod 4$, then $\pi_{n}\left(S O_{n+1}\right)=Z+Z$, generated by $\tau$ and $\sigma . \pi^{*} j^{*}$ is $1-1$, hence $j^{*}$ is $1-1$. Thus $\left[P^{n}, S O_{n+1}\right]$ contains $Z+Z$. One of the generators is $\tau_{p}$, since $\pi^{*} \tau_{p}=\tau_{p} \pi=\tau$. Therefore $j^{*} \tau=2 \tau_{p}$. For $m \equiv 2 \bmod 4, \pi_{m}\left(S O_{m+2}\right)$ $=0$ and $\left[P^{m}, S O_{m+2}\right]=K O^{-1}\left(P^{m}\right)=Z_{2}$ by (3.5). Hence, the exact sequence (3.6) becomes $0 \rightarrow Z+Z \stackrel{i^{*}}{\rightarrow}\left[P^{n}, S O_{n+1}\right] \rightarrow Z_{2} \rightarrow 0$. Since $j^{*} \tau=2 \tau_{p}$, we see that $\left[P^{n}, S O_{n+1}\right]=Z+Z$, generated by $\tau_{p}$ and $b=j^{*} \sigma . \pi^{*} b=\pi^{*} j^{*} \sigma$ $=2 \sigma$.

If $n \equiv 5 \bmod 8$, then $\pi_{n}\left(S O_{n+1}\right)=Z$, generated by $\tau$. The argument in the preceding paragraph shows that $\left[P^{n}, S O_{n+1}\right]=Z$, generated by $\tau_{p}$, and $j^{*} \tau=2 \tau_{p}$.

Now consider the following commutative diagram, where the rows are Puppe sequences and the columns are fibre mapping sequences.

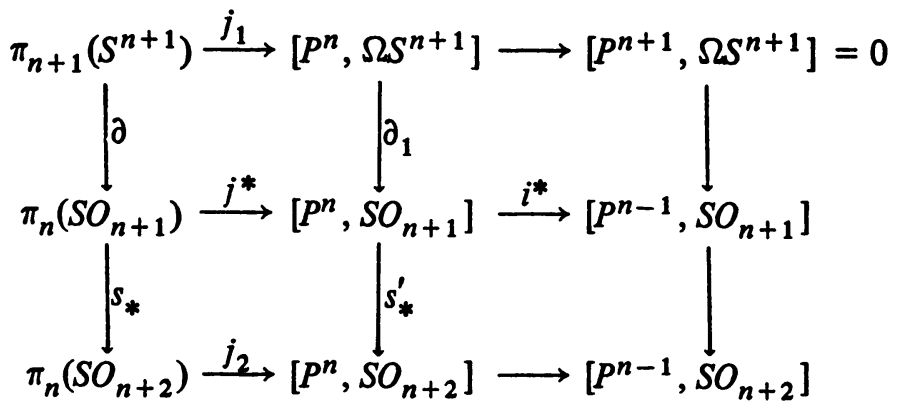

If $n \equiv 1 \bmod 8$, then $\pi_{n}\left(S O_{n+1}\right)=Z+Z_{2}$, generated by $\tau$ and $\sigma$ respectively. Since $j^{*}$ is $1-1$ on the $Z$ summand, and $\pi^{*} \tau_{p}=\tau,\left[P^{n}, S O_{n+1}\right]$ contains a $Z$ subgroup which is generated by $\tau_{p}$. Let $\iota_{n+1}$ denote the generator of $\pi_{n+1}\left(S^{n+1}\right)$. In the diagram (3.7), $\partial_{n+1}=\tau$ and $\left[P^{n}, \Omega S^{n+1}\right]=\left[\Sigma P^{n}, S^{n+1}\right]$ $=H^{n}\left(P^{n}\right)=Z$ is generated by $j_{1} \iota_{n+1} \cdot\left[P^{n}, S O_{n+2}\right]=K O^{-1}\left(P^{n}\right)=Z_{2}$ by (3.5). The middle column of (3.7) reads $Z \stackrel{\partial_{1}}{\rightarrow}\left[P^{n}, S O_{n+1}\right] \stackrel{s^{\prime}}{\rightarrow} Z_{2}$. If $\partial_{1}\left(a j_{1} \iota_{n+1}\right)=\tau_{p}$ for some integer $a$, then $j^{*}(a \tau)=a j^{*} \partial_{n+1}=\partial_{1}\left(a j_{1} \iota_{n+1}\right)=\tau_{p}$, a contradiction. Hence $\tau_{p} \in$ image $\partial_{1}, s_{*}^{\prime} \tau_{p} \neq 0$. $\left[P^{n}, S O_{n+1}\right]=Z$, generated by $\tau_{p}$. Also $j^{*} \tau=2 \tau_{p}, j^{*} \sigma=0$.

We now consider the case $n$ is even.

LEMMA 3.8. For $n$ even, $\left[P^{n}, S O_{n+1}\right]=Z_{2}$, which is generated by $\tau_{p}$.

Proof. In the diagram (3.7), $j_{1}$ is onto. Let $\iota_{n+1}$ generate $\pi_{n+1}\left(S^{n+1}\right)$. $j_{1} \iota_{n+1}$ generates $\left[P^{n}, \Omega S^{n+1}\right]=H^{n+1}\left(\Sigma P^{n}\right)=Z_{2} \cdot \partial_{1}\left(j_{1} \iota_{n+1}\right)=j^{*} \partial_{n+1}=$ $j^{*} \tau=\tau j=0$ by (3.3). The map $s_{*}^{\prime}$ in the middle column in (3.7) is onto by (3.4). Hence $s_{*}^{\prime}:\left[P^{n}, S O_{n+1}\right] \rightarrow\left[P^{n}, S O_{n+2}\right]=K O^{-1}\left(P^{n}\right)=Z_{2}$ is an isomorphism. Thus $\left[P^{n}, S O_{n+1}\right]=Z_{2}$, generated by $\tau_{p}$. Q.E.D.

Summing up, we have the following: 
THeOREM 3.9. Assume $n \neq 1,2,3,7$. For $n$ even, $\left[P^{n}, S O_{n+1}\right]=Z_{2}$, generated by $\tau_{p}$, and $\pi^{*} \tau_{p}=\tau \in \pi_{n}\left(S O_{n+1}\right)$. For $n \equiv 1 \bmod 4,\left[P^{n}, S O_{n+1}\right]$ $=Z$, generated by $\tau_{p}$, and $\pi^{*} \tau_{p}=\tau$. For $n \equiv 3 \bmod 4,\left[P^{n}, S O_{n+1}\right]=Z+Z$, generated by $\tau_{p}$ and $b$, where $b=j^{*} \sigma$, and $\pi^{*} \tau_{p}=\tau, \pi^{*} b=2 \sigma$.

Let $\operatorname{Im} \pi^{*}$ denote the image of $\left[P^{n}, S O_{n+1}\right]$ in $\pi_{n}\left(S O_{n+1}\right)$ under $\pi^{*}$.

$$
\begin{array}{ccccccccc}
n(\bmod 8) & 0 & 1 & 2 & 3 & 4 & 5 & 6 & 7 \\
\pi_{n}\left(S O_{n+1}\right) & Z_{2}+Z_{2} & Z+Z_{2} & Z_{2} & Z+Z & Z_{2} & Z & Z_{2} & Z+Z \\
& \tau, \sigma & \tau, \sigma & \tau & \tau, \sigma & \tau & \tau & \tau & \tau, \sigma \\
\operatorname{Im} \pi^{*} & \tau & \tau & \tau & \tau, 2 \sigma & \tau & \tau & \tau & \tau, 2 \sigma
\end{array}
$$

COROLlaRY 3.10. $n>3$ and $\neq 7$. Let $s_{*}^{\prime}:\left[P^{n}, S O_{n+1}\right] \rightarrow\left[P^{n}, S O_{n+2}\right]$ $=K O^{-1}\left(P^{n}\right)$ be induced by the inclusion. Then $s_{*}^{\prime}\left(\tau_{p}\right)$ generates the $Z_{2}$ summand of $K O^{-1}\left(P^{n}\right)$; and for $n \equiv 3 \bmod 4, s_{*}^{\prime}(b)$ generates the $Z$ summand of $K \mathrm{O}^{-1}\left(P^{n}\right)$.

Proof. We have proved the corollary for $n$ even in (3.8). For $n$ odd, $s_{*}^{\prime}$ : $\left[P^{n}, S O_{n+1}\right] \rightarrow\left[P^{n}, S O_{n+2}\right]$ is onto, (3.4). In (3.7), let $\iota_{n+1}$ generate $\pi_{n+1}\left(S^{n+1}\right), j_{1} \iota_{n+1}$ generates $\left[P^{n}, \Omega S^{n+1}\right] . \partial_{1}\left(j_{1} \iota_{n+1}\right)=j^{*} \partial_{n+1}=j^{*} \tau=$ $2 \tau_{p}$. Hence $s_{*}^{\prime}$ maps $\tau_{p}$ to the generator of the $Z_{2}$ summand of $K O^{-1}\left(P^{n}\right)$, and maps $b$ to the generator of the $Z$ summand for $n \equiv 3 \bmod 4$ by the exactness of the middle column in (3.7). Q.E.D.

4. Proof of Proposition 1.2. We are going to prove Proposition 1.2 in this section. Let $D\left(S^{n} \times S^{n}\right)\left(D\left(S^{n} \times S^{n}, A\right)\right)$ denote the group of concordance (A-concordance) classes of diffeomorphisms ( $A$-diffeomorphisms) of $S^{n} \times S^{n}$. $\varnothing: D\left(S^{n} \times S^{n}, A\right) \rightarrow D\left(S^{n} \times S^{n}\right)$ is the homomorphism forgetting the action. Define $D_{0}\left(S^{n} \times S^{n}\right)=$ the subgroup of $D\left(S^{n} \times S^{n}\right)$ consisting of those elements which are homotopic to Id. Let $J_{2 n+1}=\varnothing^{-1}\left(D_{0}\left(S^{n} \times S^{n}\right)\right)$. Given a diffeomorphism (an $A$-diffeomorphism) $f$ of $S^{n} \times S^{n}$, we will write $\{f\}\left(\{f\}_{A}\right)$ for its concordance class in $D\left(S^{n} \times S^{n}\right)\left(D\left(S^{n} \times S^{n}, A\right)\right)$.

If $f$ is a diffeomorphism of $S^{n} \times S^{n}$, then $f_{*}$ induces an automorphism of $H_{n}\left(S^{n} \times S^{n}\right)$. We can associate to $f_{*}$ its matrix representative $M_{f}$ with respect to the natural basis $\left\{S^{n} \times 0,0 \times S^{n}\right\}$ of $H_{n}\left(S^{n} \times S^{n}\right) . M_{f}$ is an element of $G L(2, Z)$ the group of $2 \times 2$-unimodular matrices. Let $\psi: D\left(S^{n} \times S^{n}\right) \rightarrow G L(2, Z)$ be the homomorphism defined by $f \rightarrow M_{f}$. We have $f g \rightarrow M_{g} \cdot M_{f}$.

From [19], we have the following:

Proposition 4.1 [19]. If $n$ is even, then image $\psi$ consists of eight matrices: 
$\left(\begin{array}{cc} \pm 1 & 0 \\ 0 & \pm 1\end{array}\right)$ and $\left(\begin{array}{cc}0 & \pm 1 \\ \pm 1 & 0\end{array}\right)$. If $n=1,3,7$, then image $\psi=G L(2, Z)$. If $n$ is odd, but $n \neq 1,3,7$, then image $\psi$ is the subgroup of $G L(2, Z)$ consisting of matrices $\left(\begin{array}{cc}a & b \\ c & d\end{array}\right)$ where $a b \equiv c d \equiv 0 \bmod 2$.

LEMMA 4.2. If $f$ is a diffeomorphism of $S^{n} \times S^{n}$ such that $S^{n} \times D^{n+1} U_{f}$ $D^{n+1} \times S^{n}$ is a homotopy sphere, then $M_{f}=\left(\begin{array}{ll}a & b \\ c & \pm 1\end{array}\right)$.

Proof. Write $V_{1}=S^{n} \times D^{n+1}, V_{2}=D^{n+1} \times S^{n}, \partial V_{1}=\partial V_{2}=S^{n} \times S^{n}$. Let $i_{k}: \partial V_{k} \rightarrow V_{k}, k=1$ or 2 , be the inclusion. Let $\{x, y\}$ denote the natural basis $\left\{S^{n} \times 0,0 \times S^{n}\right\}$ of $H_{n}\left(S^{n} \times S^{n}\right)$, and $u_{1}, u_{2}$ the generator of $H_{n}\left(S^{n} \times D^{n+1}\right)$, $H_{n}\left(D^{n+1} \times S^{n}\right)$ respectively. From the Mayer-Vietoris sequence, we have

$$
0 \longrightarrow H_{n}\left(\partial V_{1}\right) \stackrel{\left(j_{1}, j_{2}\right)}{\longrightarrow} H_{n}\left(V_{1}\right) \oplus H_{n}\left(V_{2}\right) \longrightarrow 0
$$

where $j_{1}=i_{1 *}$ and $j_{2}=i_{2 *} f_{*} \cdot i_{1 *} x=u_{1}, i_{1 *} y=0, i_{2 *} x=0, i_{2 *} y=u_{2}$. Let $M_{f}=\left(\begin{array}{cc}a & b \\ c & d\end{array}\right) . f_{*} x=a x+b y$ and $f_{*} y=c x+d y$. Hence $j_{1} x=u_{1}, j_{1} y=0$, $j_{2} x=i_{2 *} f_{*} x=b u_{2}, j_{2} y=i_{2 *} f_{*} y=d u_{2}$. Thus the matrix for $\left(j_{1}, j_{2}\right)$ with respect to the basis $\{x, y\}$ and $\left\{u_{1}, u_{2}\right\}$ is $\left(\begin{array}{ll}1 & b \\ 0 & b\end{array}\right)$, which is unimodular, $d= \pm 1$. Q.E.D.

LEMMA 4.3. $n>3$ and $\neq 7$. If $\left(\Sigma^{2 n+1}, T\right)=\left(\Sigma_{f}, T_{f}\right)$ for some A-diffeomorphism $f$ of $S^{n} \times S^{n}$, then there exists another Addiffeomorphism $g$ such that $M_{g}$ is the identity matrix, and $\left(\Sigma^{2 n+1}, T\right)=\left(\Sigma_{g}, T_{g}\right)$.

Proof. If $n$ is even, then it follows from (4.1) and (4.2) that $M_{f}=\left(\begin{array}{cc} \pm 1 & 0 \\ 0 & \pm 1\end{array}\right)$. Consider the $A$-diffeomorphisms $h_{1}$ and $h_{2}$ of $S^{n} \times S^{n}$ defined by $h_{1}(x, y)=$ $(x,-y)$ and $h_{2}(x, y)=(-x, y) . h_{1}$ (or $h_{2}$ ) extends equivariantly to $\left(S^{n} \times D^{n+1}, A\right)$ (or $\left(D^{n+1} \times S^{n}, A\right)$ ). One of the $A$-diffeomorphisms $f, f h_{1}$, $h_{2} f h_{1}$, or $h_{2} f$ has the corresponding matrix $=$ identity matrix. Take $g$ to be this map. Also, $\left(\Sigma^{2 n+1}, T\right)=\left(\Sigma_{g}, T_{g}\right)$ by (2.1).

If $n$ is odd and $\neq 1,3,7$, then $M_{f}=\left(\begin{array}{ll}a & b \\ c \pm 1\end{array}\right)$ by (4.2). We can compose $f$ with $h_{1}$ or $h_{2}$ if necessary, to make $M_{f}=\left(\begin{array}{ll}a & b \\ c & 1\end{array}\right)$ and det $M_{f}=+1$. From (4.1), $b \equiv$

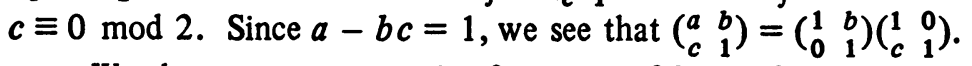

We choose a representative for $\tau$ as in $\S 3$, satisfying $\tau(x)=\tau(-x)$. Consider the maps $f_{\tau}$ and $g_{\tau}$, defined by $f_{\tau}(x, y)=(x, \tau(x) \cdot y)$ and $g_{\tau}(x, y)=(\tau(y) \cdot x, y)$. $f_{\tau}$ and $g_{\tau}$ are $A$-diffeomorphisms. Theorem 7.10.1 of [14] showed that the map $q \circ \tau$ in (3.2) is of degree 2. Hence $M_{f_{\tau}}=\left(\begin{array}{ll}1 & 2 \\ 0 & 1\end{array}\right)$ and $M_{g_{\tau}}=\left(\begin{array}{ll}1 & 0 \\ 2 & 1\end{array}\right)$. Define $g=$ $g_{\tau}^{(-c / 2)} f f_{\tau}^{(-b / 2)}$. Since $M_{f g}=M_{g} M_{f}$, we see that $M_{g}$ is the identity matrix. $\left(\Sigma_{f}, T_{f}\right)=\left(\Sigma_{g}, T_{g}\right)$ by $(2.1)$. Q.E.D.

Lemma 4.4. A diffeomorphism $f$ of $S^{n} \times S^{n}$ is homotopic to Id if and only if $M_{f}$ is the identity matrix. 
Proof. If $f$ and $g$ are diffeomorphisms of $S^{n} \times S^{n}$ such that $\{f\}=\{g\}$ in $D\left(S^{n} \times S^{n}\right)$, then $M_{f}=M_{g}$. Let $G=\left\{\{f\} \in D\left(S^{n} \times S^{n}\right) \mid M_{f}\right.$ is the identity matrix $\}$. Theorem II of [28] showed that $0 \rightarrow H_{1}+\Gamma^{2 n+1} \rightarrow G \rightarrow H_{2} \rightarrow 0$ is exact, where $H_{i}, i=1$ or 2 , is isomorphic to image $\left\{s_{*}: \pi_{n}\left(S O_{n}\right) \rightarrow \pi_{n}\left(S O_{n+1}\right)\right\}$. Let $a$ be a smooth map representing $\{a\} \in$ image $s_{*} \subseteq \pi_{n}\left(S O_{n+1}\right)$. We define two diffeomorphisms $f_{a}$ and $g_{a}$ of $S^{n} \times S^{n}$ by $f_{a}(x, y)=(x, a(x) \cdot y)$ and $g_{a}(x, y)=$ $(a(y) \cdot x, y)$. The maps $\{a\} \rightarrow\left\{f_{a}\right\}$ and $\{a\} \rightarrow\left\{g_{a}\right\}$ are isomorphisms of image $s_{*}$ to $H_{1}$ and $H_{2}$ respectively, (compare 2.2). $\Gamma^{2 n+1}$ is the Kervaire-Milnor group [17] and acts by leaving the complement of a $2 n$-disk in $S^{n} \times S^{n}$ fixed.

Let $a$ be a smooth map representing a homotopy class $\{a\} \in \pi_{n}\left(S O_{n+1}\right)$ such that $\{a\}=s_{*} \beta$ for some $\beta \in \pi_{n}\left(S O_{n}\right)$. We can take $a \mid D_{-}^{n}=\mathrm{Id}$, where $D_{-}^{n}\left(D_{+}^{n}\right)$ denotes the lower (upper) hemisphere of $S^{n}$. Hence $f_{a} \mid D_{-}^{n} \times S^{n}=$ Id. Let $e$ denote the north pole of $S^{n}$. Since $\{a\}=s_{*} \beta$, we can take $f_{a} \mid S^{n} \times\{e\}=$ Id. By the homotopy extension theorem, $f_{a}$ is homotopic to a map $h$ such that $h$ is the identity on a neighborhood $N$ of $S^{n} \times\{e\}$ and $f_{a}=h$ on $D_{-}^{n} \times S^{n}$. $h$ keeps

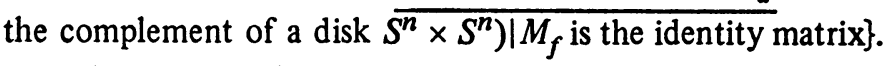

Since every element $\{h\}$ of $G$ has a representative $h$ such that $h$ leaves the complement of a $2 n$-disk fixed, we can apply the Alexander trick to see that $h$ is homotopic to Id. Thus $G=D_{0}\left(S^{n} \times S^{n}\right)$. Q.E.D.

Combining (4.3) and (4.4) together, we have proved Proposition (1.2). From now on, when $n>3$ and $\neq 7$, we will assume the $A$-diffeomorphism $f$ in the decomposition $\left(\Sigma^{2 n+1}, T\right)=\left(\Sigma_{f}, T_{f}\right)$, be homotopic to Id.

5. Group structure on $h S\left(P^{2 n+1}\right)$. Given an involution $\left(\Sigma^{2 n+1}, T\right), n>3$ and $\neq 7$, there exists an $A$-diffeomorphism $f$ of $S^{n} \times S^{n}$ such that $\left(\Sigma^{2 n+1}, T\right)=$ $\left(\Sigma_{f}, T_{f}\right)$, and $\left(\Sigma_{f}, T_{f}\right)=\left(\Sigma_{g}, T_{g}\right)$ if $g \in\{f\}_{A} \in J_{2 n+1}=\varnothing^{-1}\left(D_{0}\left(S^{n} \times S^{n}\right)\right)$. But $\{f\}_{A} \in J_{2 n+1}$ is not uniquely determined by $\left(\Sigma^{2 n+1}, T\right)$. Suppose we can find a subgroup $G_{2 n+1}$ of $J_{2 n+1}$ such that $G_{2 n+1}$ is in 1-1 correspondence with $h S\left(P^{2 n+1}\right)$ under the mapping $\{f\}_{A} \rightarrow\left(\Sigma_{f}, T_{f}\right)$. Then $\left(h S\left(P^{2 n+1}\right)\right.$, *) forms a group by carrying over the composition law in $G_{2 n+1}:\left(\Sigma_{f}, T_{f}\right)^{*}\left(\Sigma_{g}, T_{g}\right)=$ $\left(\Sigma_{f g}, T_{f g}\right)$. In this section, we will show that such a subgroup $G_{2 n+1}$ exists for $n \equiv 0,1,2 \bmod 4$.

THEOREM 5.1. For $n$ even, and $>2$, such a subgroup $G_{2 n+1}$ of $J_{2 n+1}$ exists, hence $\left(h S\left(P^{2 n+1}\right), *\right)$ is a subgroup.

Proof. From [19] or (4.4) above, we know that $D_{0}\left(S^{n} \times S^{n}\right)$ is the semidirect product of $H_{1}+\Gamma^{2 n+1}$ and $H_{2}$, where $H_{1}$ and $H_{2}$ are isomorphic to image $\left\{s_{*}: \pi_{n}\left(S O_{n}\right) \rightarrow \pi_{n}\left(S O_{n+1}\right)\right\}$, which is equal to $\pi_{n}\left(S O_{n+1}\right)$, [18]. $\pi_{n}\left(S O_{n+1}\right)=Z_{2}$, generated by $\tau$ for $n \neq 0 \bmod 8 ; \pi_{n}\left(S O_{n+1}\right)=Z_{2}+Z_{2}$, generated by $\tau$ and $\sigma$ for $n \equiv 0 \bmod 8$. Every element of $D_{0}\left(S^{n} \times S^{n}\right)$ can be 
uniquely expressed in the form $h_{2} y h_{1}$, where $h_{i} \in H_{i}$ and $y \in \Gamma^{2 n+1}$, [17]. We define a subgroup $F_{2 n+1}$ of $D_{0}\left(S^{n} \times S^{n}\right)$ as follows: $F_{2 n+1}=\Gamma^{2 n+1}$ for $n \neq 0$ $\bmod 8$; if $n \equiv 0 \bmod 8$, then $F_{2 n+1}$ is the semidirect product of $\left(\sigma_{1}\right)+\Gamma^{2 n+1}$ and $\left(\sigma_{2}\right)$, where $\left(\sigma_{i}\right)$ denotes the cyclic group of order 2 generated by $\sigma_{i}: \sigma_{1}(x, y)$ $=(x, \sigma(x) \cdot y), \sigma_{2}(x, y)=(\sigma(y) \cdot x, y)$. Let $G_{2 n+1}=\varnothing^{-1}\left(F_{2 n+1}\right)$.

We choose a smooth representative for $\tau \in \pi_{n}\left(S O_{n+1}\right)$ such that $\tau(x)=$ $\tau(-x)$. Define $\tau_{1}$ and $\tau_{2}$ by $\tau_{1}(x, y)=(x, \tau(x) \cdot y)$ and $\tau_{2}(x, y)=(\tau(y) \cdot x, y)$. $\tau_{1}$ and $\tau_{2}$ are $A$-diffeomorphisms of $S^{n} \times S^{n}$. Since $\tau$ commutes with $\sigma$ in $\pi_{n}\left(S O_{n+1}\right),[19]$, we see that any element of $D_{0}\left(S^{n} \times S^{n}\right)$ can be uniquely expressed in the form bya, where $b \in\left(\tau_{2}\right), a \in\left(\tau_{1}\right)$, and $y \in F_{2 n+1}$.

If $h \in\{h\}_{A} \in J_{2 n+1}$, then $\varnothing\left(\{h\}_{A}\right)=\left\{\tau_{2}^{d}\right\}\{f\}\left\{\tau_{1}^{c}\right\}$, where $\{f\} \in F_{2 n+1}$, and $c, d=0$ or 1 uniquely determined by $\{h\}_{A}$. We define $g=\tau_{2}^{d} h \tau_{1}^{c}$, which is an $A$-diffeomorphism of $S^{n} \times S^{n}$. Since $\left\{\tau_{i}\right\}$ is of order two in $D_{0}\left(S^{n} \times S^{n}\right)$, $\varnothing\left(\{g\}_{A}\right) \in F_{2 n+1}$. $\left(\Sigma_{g}, T_{g}\right)=\left(\Sigma_{h}, T_{h}\right)$ by (2.1). On the other hand, if $\{f\}_{A}$, $\{g\}_{A} \in G_{2 n+1}$ and $\left(\Sigma_{f}, T_{f}\right)=\left(\Sigma_{g}, T_{g}\right)$, then $g$ is $A$-concordant to $\tau_{2}^{d} f \tau_{1}^{c}$, where $c, d=0$ or 1, by (2.1) and (3.9). But $\varnothing\left(\{f\}_{A}\right), \varnothing\left(\{g\}_{A}\right) \in F_{2 n+1}$. Hence $c=$ $d=0,\{f\}_{A}=\{g\}_{A}$. Q.E.D.

THEOREM 5.2. For $n \equiv 1 \bmod 4, h S\left(P^{2 n+1}\right)$ is in $1-1$ correspondence with $J_{2 n+1}$; hence $\left(h S\left(P^{2 n+1}\right), *\right)$ forms a group.

Proof. As in (5.1), we know that $D_{0}\left(S^{n} \times S^{n}\right)$ is the semidirect product of $H_{1}+\Gamma^{2 n+1}$ and $H_{2}$, where $H_{1}$ and $H_{2}$ are isomorphic to image $\left\{s_{*}: \pi_{n}\left(S O_{n}\right)\right.$ $\left.\rightarrow \pi_{n}\left(S O_{n+1}\right)\right\}$, which is 0 for $n \equiv 5 \bmod 8$; and $Z_{2}$, generated by $\sigma$ for $n \equiv 1$ $\bmod 8$. $\sigma \notin$ image $\left\{\pi^{*}:\left[P^{n}, S O_{n+1}\right] \rightarrow\left[S^{n}, S O_{n+1}\right]\right\}$ by (3.9). Hence no element in $D_{0}\left(S^{n} \times S^{n}\right)$ is concordant to an $A$-bundle map by (2.5). Thus $h S\left(P^{2 n+1}\right)$ is in 1-1 correspondence with $J_{2 n+1}=\varnothing^{-1}\left(D_{0}\left(S^{n} \times S^{n}\right)\right)$ by (1.2) and (2.1). Q.E.D.

REMARK 5.3. For $n>7$ and $n \equiv 3 \bmod 4$, we have the exact sequence: $0 \rightarrow Z+\Gamma^{2 n+1} \rightarrow D_{0}\left(S^{n} \times S^{n}\right) \rightarrow Z \rightarrow 0$, where each $Z$ is isomorphic to image $\left\{s_{*}: \pi_{n}\left(S O_{n}\right) \rightarrow \pi_{n}\left(S O_{n+1}\right)\right\}$, generated by $\sigma$. We know that $2 m \sigma \in$ image $\pi^{*}$ but $(2 m+1) \sigma \notin$ image $\pi^{*}$ by (3.9). Let $\sigma_{1}$ and $\sigma_{2}$ be defined by $\sigma_{1}(x, y)=(x, \sigma(x) \cdot y), \sigma_{2}(x, y)=(\sigma(y) \cdot x, y)$. If none of the four diffeomorphisms $\sigma_{1}, \sigma_{2}, \sigma_{2} \sigma_{1}$, and $\sigma_{1} \sigma_{2}$ is concordant to an $A$-diffeomorphism, i.e. $\left\{\sigma_{1}\right\},\left\{\sigma_{2}\right\},\left\{\sigma_{2} \sigma_{1}\right\},\left\{\sigma_{1} \sigma_{2}\right\} \notin$ image $\varnothing$, then we can take $G_{2 n+1}=\varnothing^{-1}\left(\Gamma^{2 n+1}\right)$ as in (5.1) and (5.2).

REMARK 5.4. The case $n=3, h S\left(P^{7}\right)$. Viewing $S^{3}$ as the unit sphere in the quaternionic space, we define $\{r\},\{t\} \in \pi_{3}\left(S_{4}\right)$ by $r(x) \cdot y=x y x^{-1}, t(x)$ - $y=x y . \quad \pi_{3}\left(\mathrm{SO}_{4}\right)=Z+Z$ is generated by $\{r\}$ and $\{t\},[14$, p. 94]. As in (3.9), we can show that image $\left\{\pi^{*}:\left[P^{3}, \mathrm{SO}_{4}\right] \rightarrow \pi_{3}\left(\mathrm{SO}_{4}\right)\right\}$ is generated by $\{r\}$ and $2\{t\}$. 
Let $t_{1}$ and $t_{2}$ be defined in the same way as $\sigma_{1}$ and $\sigma_{2}$ in (5.3). If $\left\{t_{1}\right\},\left\{t_{2}\right\}$, $\left\{t_{1} t_{2}\right\},\left\{t_{2} t_{1}\right\} \notin$ image $\varnothing$, then (1.2) is also true in this case and $\left(h S\left(P^{7}\right), *\right)$ forms a group.

6. Additivity of Browder-Livesay index invariant. In [7], [8], Browder and Livesay defined a desuspension invariant $\sigma$ for any free involution $\left(\Sigma^{2 n+1}, T\right)$ as follows: construct an $(n-1)$-connected characteristic submanifold $N^{2 n}$ for $\left(\Sigma^{2 n+1}, T\right)$, i.e. $\Sigma^{2 n+1}=A \cup B, A \cap B=N, T A=B, T N=N$, and $A, B$ are $(2 n+1)$-submanifolds of $\Sigma^{2 n+1}$. Let $K_{n}=\operatorname{Ker}\left(H_{n}(N) \rightarrow H_{n}(A)\right)$. If $n$ is odd, they define a unimodular even symmetric bilinear form $B$ on $K_{n}$ (modulo torsion) by $B(x, y)=x \cdot T_{*} y$. Let $\sigma\left(\Sigma^{2 n+1}, T\right)=(1 / 8)$ index $B \in Z$. If $n$ is even, they use $Z_{2}$ as coefficients and define the unimodular bilinear form $B_{2}$ on $K_{n}$ (with $Z_{2}$ coefficients) by $B_{2}(x, y)=x \cdot T_{*} y$. They also defined a quadratic form $\psi: K_{n} \rightarrow Z_{2}$ associated to $B_{2}, B_{2}(x, y)=\psi(x+y)+\psi(x)+\psi(y)$, such that, if $x \in K_{n}$ is represented by an immersed sphere $d$, then $\psi(x)=1$ iff $d \cap T d$ in general position consists of an odd number of pairs of points. Write $\sigma\left(\Sigma^{2 n+1}, T\right)$ $=c(\psi) \in Z_{2}$, the Art invariant of $\psi$. They also showed that for $n \geqslant 3$, $\sigma\left(\Sigma^{2 n+1}, T\right)=0$ iff $\left(\Sigma^{2 n+1}, T\right)$ admits a codimension 1 invariant sphere (for details, see [8]).

From now on, we assume $n \geqslant 3$. Suppose $\left(\Sigma^{2 n+1}, T\right)=\left(\Sigma_{f}, T_{f}\right)$ for an $A$-diffeomorphism $f$ of $S^{n} \times S^{n}$, let $j: S^{n} \times S^{n} / A \rightarrow S^{n} \times S^{m-1} / A \rightarrow P^{n+m}=$ $\left(S^{n} \times D^{m} / A\right) \cup_{\mathrm{Id}}\left(D^{n+1} \times S^{m-1} / A\right)$, with $m$ large, be the natural inclusion.

Both $j \circ(f / A)$ and $j$ are classifying maps for the same $Z_{2}$-bundle. Hence they are homotopic, by a map $F:\left(S^{n} \times S^{n} \times I\right) / A \times \mathrm{Id} \rightarrow P^{n+m}$. We may suppose that $F$ is smooth and transverse regular on $P^{n+m-1}=\left(S^{n} \times D^{m-1} / A\right) \cup_{\mathrm{Id}}\left(D^{n+1} \times\right.$ $\left.S^{m-2} / A\right)$, relative boundary. The double cover $M$ of $F^{-1}\left(P^{n+m-1}\right)$ is a characteristic submanifold of $\left(S^{n} \times S^{n} \times I, A \times\right.$ Id), with $\partial M=M_{1}-M_{0}$, where $M_{1}=$ $f^{-1}\left(S^{n} \times S^{n-1}\right) \times 1, M_{0}=\left(S^{n} \times S^{n-1}\right) \times 0$. We recall that a codim 1 characteristic submanifold $M$ of a free involution $(W, T)$ is a codim 1 submanifold of $W$ such that $W=A \cup B$, where $A$ and $B$ are codim 0 submanifolds of $W, A \cap B=M$, and $T A=B$, [8].

In the rest of this section, we will write $(W, T)=\left(S^{n} \times S^{n} \times I, A \times\right.$ Id), $W_{1}=S^{n} \times S^{n} \times 1, W_{0}=S^{n} \times S^{n} \times 0$; also, let $W=V \cup T V, V \cap T V=M$, $V_{i}=V \cap W_{i}$ for $i=0,1$, where $V_{0}=S^{n} \times D^{n}, V_{1}=f^{-1}\left(S^{n} \times D^{n}\right)$.

Since $S^{n} \times S^{n} / A$ is the total space of a spherical fibre bundle $S^{n} \rightarrow S^{n} \times$ $S^{n} / A \rightarrow S^{n} / A,(\S 1)$. By Gysin sequence, $H_{k}\left(S^{n} \times S^{n} / A ; Z_{2}\right)=Z_{2}$ for $k \neq n$, and $=Z_{2}+Z_{2}$ for $k=n$. Hence $H_{k}\left(S^{n} \times S^{n} / A ; Z_{2}\right)=H_{k}\left(W / T ; Z_{2}\right)=$ $H^{2 n+1-k}\left(W / T, \partial W / T ; Z_{2}\right)$.

We want to make a characteristic submanifold $M$ of $(W, T)$ as highly connected as possible. 
LEMMA 6.1. There exists a connected characteristic submanifold $M$ for $(W, T)$ with $\partial M=M_{1}-M_{0}$.

Proof. Let $M$ be a characteristic submanifold constructed above with $\partial M=$ $M_{1}-M_{0}$. Then $(M, \partial M) / T$ carries the unique nonzero element of $H_{2 n}\left(W / T, \partial W / T, Z_{2}\right)$ $=Z_{2}$, dual to the 1 -dimensional cohomology class $F^{*} x$, where $x$ generates $H^{1}\left(P^{n+m} ; Z_{2}\right)$ and $F$ is the classifying map constructed above, [32]. Hence a component of $M / T$ carries this element. Let $M^{\prime}$ be the double cover of this component. If $M^{\prime} \cap M_{0}=\varnothing$, then we can take $y \in H^{1}\left(W_{0} / T ; Z_{2}\right)=H^{1}\left(W / T ; Z_{2}\right)$ $=Z_{2}$ representing $F^{*} x$, hence $H_{2 n}\left(M^{\prime} / T, \partial M^{\prime} / T ; Z_{2}\right) \rightarrow H_{2 n}\left(W / T, \partial W / T ; Z_{2}\right)$ is trivial, a contradiction. Hence $M_{0} \cap M^{\prime} \neq \varnothing$. Since $M_{0}$ is a closed connected manifold, $M_{0} \subseteq M^{\prime}$. Similarly $M_{1} \subseteq M^{\prime}$. It is clear that $T$ interchanges the two components of $W-M^{\prime}$, so that $M^{\prime}$ is a characteristic submanifold. We will write $M$ for $M^{\prime}$. Q.E.D.

LEMMA 6.2. There exists a simply connected characteristic submanifold $M$ for $(W, T)$ with $\partial M=M_{1}-M_{0}$.

Proof. Since $\operatorname{dim} W=2 n+1 \geqslant 7$ and $\pi_{j}(W)=0$ for $j \leqslant 2$, the proof is exactly the same as in Lemma 2.2 of [8] by applying [4]: We apply equivalent handle exchanges in the interior of $W$ to make $M$ 1-connected. Q.E.D.

From now on, we assume the characteristic submanifold $M$ is 1-connected, with $\partial M=M_{1}-M_{0}$. Consider the following diagram of exact sequences:

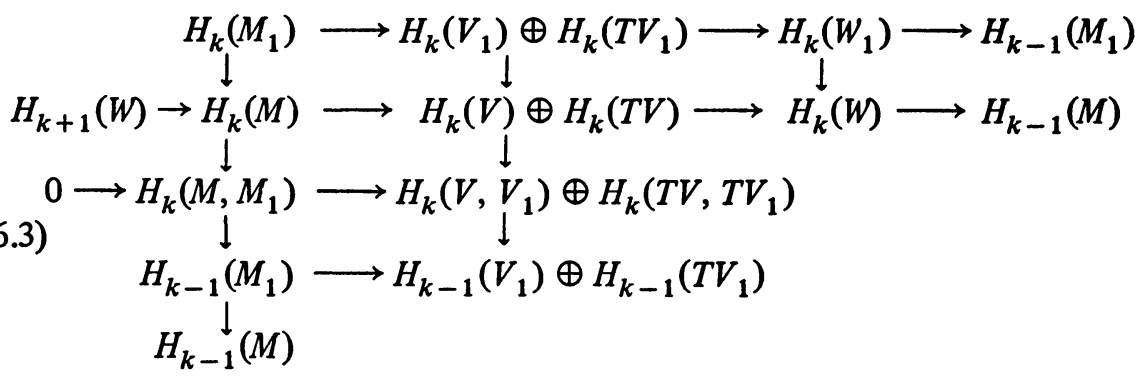

We can replace $M_{1}, V_{1}$ by $M_{0}, V_{0}$ in (6.3).

LEMMA 6.4. By performing equivariant surgery (equivariant handle exchanges) in the interior of $W$, we can transform $M$ into an $(n-2)$-connected $2 n$-characteristic submanifold. We also have $\pi_{k}\left(M, M_{1}\right)=\pi_{k}\left(M, M_{0}\right)=\pi_{k}\left(V, V_{1}\right)=\pi_{k}\left(V, V_{0}\right)$ $=0$ for $k \leqslant n-2$.

Proof. Suppose $M$ is already $(k-1)$-connected, $k-1<n-2$. From (6.3), we have 


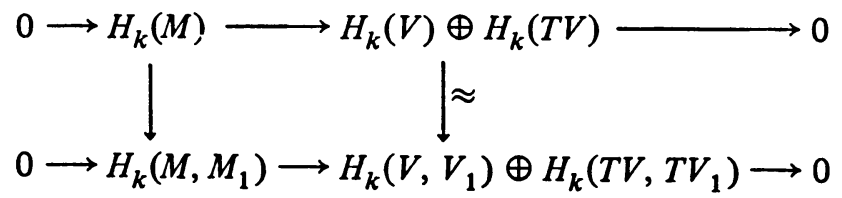

Using the first exact sequence, we can perform equivariant handle exchanges in the interior of $W$ to kill $H_{k}(M)$ as in [8], (see [8, 2.3] for details). The other part of the lemma follows from the above diagram, the induction hypothesis, and the Hurewicz theorem. Q.E.D.

From now on, we will assume $M$ to be $(n-2)$-connected. Letting $k=n-1$ in (6.3), we have

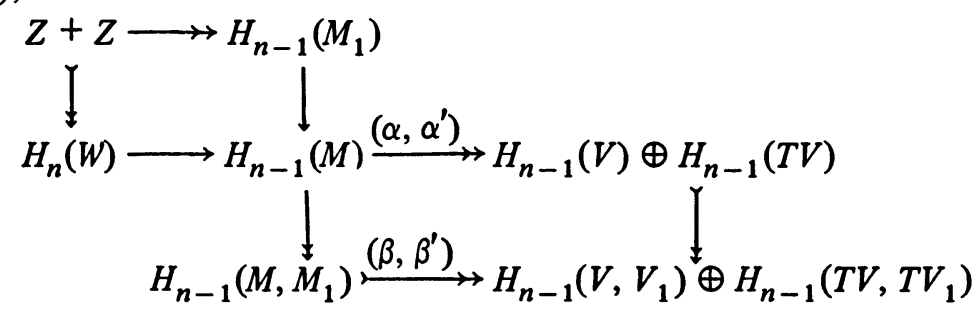

where " $\longrightarrow$ " means $1-1$, " $\rightarrow$ " onto, and " $\mapsto$ " isomorphic; $\alpha, \alpha$, $\beta, \beta$ ' are maps induced by inclusions.

As in $[8,2.3]$, we see that $H_{n-1}\left(M, M_{1}\right)=\operatorname{Ker} \beta \oplus \operatorname{Ker} \beta^{\prime}, T_{*}(\operatorname{Ker} \beta)=$ $\operatorname{Ker} \beta^{\prime}$, both $\beta: \operatorname{Ker} \beta^{\prime} \rightarrow H_{n-1}\left(V, V_{1}\right)$ and $\beta^{\prime}: \operatorname{Ker} \beta \rightarrow H_{n-1}\left(T V, T V_{1}\right)$ are isomorphisms. Similarly, $H_{n-1}(M)=\left\{a+b \mid a \in \operatorname{Ker} \alpha, b \in \operatorname{Ker} \alpha^{\prime}\right\}$ with

$$
\begin{aligned}
\operatorname{Ker} \alpha \cap \operatorname{Ker} \alpha^{\prime} & =\operatorname{Image}\left(H_{n}(W) \rightarrow H_{n-1}(M)\right) \\
& =\operatorname{Image}\left(H_{n-1}\left(M_{1}\right) \rightarrow H_{n-1}(M)\right)=\{a\},
\end{aligned}
$$

a cyclic group. Suppose $a=p x$, for some integer $P$, where $\{x\}$ is a direct summand of $H_{n-1}(M)$. Since both $\alpha=\operatorname{Ker} \alpha^{\prime} \rightarrow H_{n-1}(V)$ and $\alpha^{\prime}: \operatorname{Ker} \alpha \rightarrow$ $H_{n-1}(T V)$ are onto with kernel $=\{a\}$, if $p \neq 0$ or 1 , then $x$ contributes two copies of $Z_{p}$ in $H_{n-1}(V) \oplus H_{n-1}(T V)$, which is impossible by a simple counting argument. Hence $\operatorname{Ker} \alpha \cap \operatorname{Ker} \alpha^{\prime}=\{a\}$ is a direct summand of $H_{n-1}(M) \cdot$ $H_{n-1}(M)=\{a\}+H$, and $H \mapsto H_{n-1}(V) \oplus H_{n-1}(T V)$. We can perform equivariant handle exchanges in the interior of $W$ to kill $H$ as in $[8,2.3]$. Thus we have the following:

LEMMA 6.6. By equivariant handle exchanges in the interior of $W$, we can make $M(n-2)$-connected, with $H_{n-1}(M)$ a cyclic group; and $\pi_{k}\left(M, M_{1}\right)=$ $\pi_{k}\left(M, M_{0}\right)=0$ for $k<n$.

In the rest of this section, all homology will be taken with rational coefficient $Q$, except where explicitly stated.

Letting $k=n$ in (6.3), we have 


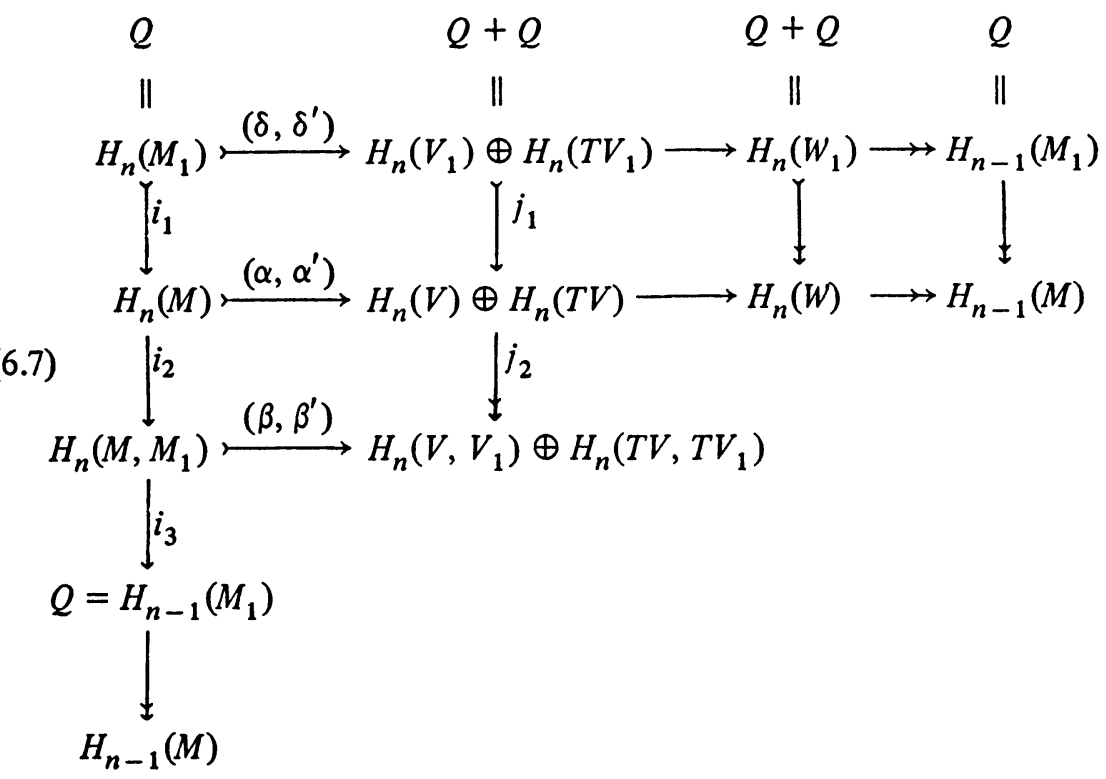

where $M$ is the characteristic submanifold for $(W, T)$ as in (6.6).

Lemma 6.8. In the diagram (6.7), $i_{2}$ maps $\operatorname{Ker} \alpha$ into $\operatorname{Ker} \beta$ injectively.

PROof. $i_{2}$ maps Ker $\alpha$ into Ker $\beta$ by the commutativity of (6.7). $H_{n}\left(M_{1}\right)$ $\rightarrow H_{n}\left(V_{1}\right)$ is an isomorphism. Since $H_{n}\left(V_{1}\right) \rightarrow H_{n}(T V)$ is trivial, $H_{n}\left(V_{1}\right) \rightarrow$ $H_{n}(V)$ is 1-1. But $\alpha i_{1}=j_{1} \delta$ from (6.7). Hence $\operatorname{Ker} \alpha \cap \operatorname{Im} i_{1}=0$. Q.E.D.

Lemma 6.9. In the diagram (6.7), if $x \in \operatorname{Ker} \beta \cap \operatorname{Ker} i_{3}$, then there exists $y \in \operatorname{Ker} \alpha$ such that $i_{2}(y)=x$.

Proof. By exactness, there exists $w \in H_{n}(M)$ such that $i_{2}(w)=x$. Let $\alpha(w)=t, j_{2}(t)=j_{2} \alpha(w)=\beta i_{2}(w)=0$. Hence there is a $z \in H_{n}\left(V_{1}\right)$ such that $j_{1}(z)=t$. But $\delta: H_{n}\left(M_{1}\right) \rightarrow H_{n}\left(V_{1}\right)$ is an isomorphism. Define $y=w-$ $i_{1} \delta^{-1}(z)$. We have $\alpha(y)=0$ and $i_{2}(y)=x$. Q.E.D.

In the diagram (6.7), $H_{n-1}(M)=Q$ or 0 by (6.6).

LEMMA 6.10. Let $m=\operatorname{rank} H_{n}\left(V, V_{1}\right)$ in the diagram (6.7)

(a) If $H_{n-1}(M)=Q$, then rank $H_{n}(M)=2 m+1$, rank Ker $\alpha=m$.

(b) If $H_{n-1}(M)=0$, then rank $H_{n}(M)=2 m$, rank Ker $\alpha=m-1$.

Proof. (a) If $H_{n-1}(M)=Q$, then $H_{n-1}\left(M_{1}\right) \rightarrow H_{n-1}(M)$ is an isomorphism. Since $\operatorname{rank} H_{n}\left(M, M_{1}\right)=2$ rank $\beta=2 m$, rank $H_{n}(M)=2 m+1$. Hence rank $\operatorname{Ker} \alpha=\operatorname{rank} \operatorname{Ker} \alpha^{\prime} \leqslant m$. But $i_{3}=0$. rank Ker $\alpha \geqslant \operatorname{rank} \operatorname{Ker} \beta=m$ by (6.8) and (6.9). Thus rank Ker $\alpha=m$.

(b) If $H_{n-1}(M)=0$, then $i_{3}$ is onto. Since rank $H_{n}\left(M, M_{1}\right)=2 m$ as in (a), rank $H_{n}(M)=2 m$. Hence rank Ker $\alpha \leqslant m-1$ from (6.8). But rank Ker $\alpha \geqslant$ $m-1$ by (6.8) and (6.9). Thus rank $\operatorname{Ker} \alpha=m-1$. Q.E.D. 
Given such a characteristic submanifold $M$ for $(W, T)$ as in (6.6), we can define a bilinear form $C_{M}^{f}$ on $H_{n}(M)$ by $C_{M}^{f}(x, y)=x \cdot T_{*} y$. Let $B_{M}^{f}=$ $C_{M}^{f} \mid \operatorname{Ker} \alpha$, where $\left(\alpha, \alpha^{\prime}\right): H_{n}(M) \rightarrow H_{n}(V) \oplus H_{n}(T V)$ is the map induced by inclusion as in (6.7).

Now, we assume $n$ odd in the rest of this section, hence $C_{M}^{f}$ is symmetric.

Lemma 6.11. index $C_{M}^{f}=2$ index $B_{M}^{f}$.

PRoof. There are two cases: (a) $H_{n-1}(M)=Q \cdot H_{n}(M)=\operatorname{Ker} \alpha \oplus \operatorname{Ker} \alpha^{\prime} \oplus$ $\operatorname{Im} i_{1}$ by (6.8) and (6.10). For $x \in H_{n}(M)$, let $\bar{x} \in H^{n}(M, \partial M)$ denote its Poincaré dual. If $x, y \in \operatorname{Ker} \alpha$, then $\bar{x}, \bar{y} \in \operatorname{Image}\left\{\alpha^{*}: H^{n}(V, \partial V) \rightarrow H^{n}(M, \partial M)\right\}$. Let $\bar{x}=\alpha^{*} u, \bar{y}=\alpha^{*} V . x \cdot y=\left\langle\alpha^{*} u \cup \alpha^{*} v,[M]\right\rangle=\left\langle u \cup v, \alpha_{*}[M]\right\rangle=\langle u \cup v, 0\rangle$ $=0$. Similarly, $x \cdot y=0$ for $x, y \in \operatorname{Ker} \alpha^{\prime}$. Since $\operatorname{Im} i_{1} \subseteq \operatorname{Image}\left\{i: H_{n}(\partial M) \rightarrow H_{n}(M)\right\}$, we see that $x \cdot z=0$ for $x \in H_{n}(M)$ and $z \in \operatorname{Im} i_{1}$. We have $T_{*} \operatorname{Ker} \alpha=\operatorname{Ker} \alpha^{\prime}$, $T_{*} \operatorname{Ker} \alpha^{\prime}=\operatorname{Ker} \alpha$, and $T_{*} \operatorname{Im} i_{1}=\operatorname{Im} i_{1}$. Hence $C_{M}^{f}(x, y)=0$ for $x \in \operatorname{Ker} \alpha$, $y \in \operatorname{Ker} \alpha^{\prime}$; or $x \in \operatorname{Im} i_{1} ; y \in H_{n}(M)$. Thus $C_{M}^{f}=C_{M}^{f}\left|\operatorname{Ker} \alpha+C_{M}^{f}\right| \operatorname{Ker} \alpha^{\prime}+$ a 1-dim trivial form. But $C_{M}^{f}\left|\operatorname{Ker} \alpha=C_{M}^{f}\right| \operatorname{Ker} \alpha^{\prime}=B_{M}^{f}$. Hence index $C_{M}^{f}=2$ index $B_{M}^{f}$.

(b) $H_{n-1}(M)=0$. Let $i: H_{n}(\partial M) \rightarrow H_{n}(M)$ be induced by the inclusion. $i$ is injective, because $H_{n+1}(M, \partial M) \cong H^{n-1}(M) \cong H_{n-1}(M)=0 . H_{n}(\partial M)=$ $H_{n}\left(M_{0}\right) \oplus H_{n}\left(M_{1}\right)=Q+Q$. The proof of (6.8) shows that

$\operatorname{Im} i \cap\left(\operatorname{Ker} \alpha \oplus \operatorname{Ker} \alpha^{\prime}\right)=0$.

Hence $H_{n}(M)=\operatorname{Ker} \alpha \oplus \operatorname{Ker} \alpha^{\prime} \oplus \operatorname{Im} i$ by (6.10). As in the case (a), we can show that $C_{M}^{f}=C_{M}^{f}\left|\operatorname{Ker} \alpha+C_{M}^{f}\right| \operatorname{Ker} \alpha^{\prime}+a 2$-dim trivial form. Hence index $C_{M}^{f}=2$ index $B_{M}^{f}$. Q.E.D.

Let $S$ be an involution on a manifold $X^{2 p}$. Let $B^{\prime}$ denote the bilinear form defined on $H_{p}\left(X^{2 p}\right)$ by $B^{\prime}(x, y)=x \cdot S_{*} y$. If $B^{\prime}$ is symmetric, define $\sigma_{s}(X)=$ index $B^{\prime}$. We need the following theorem from [31].

THEOREM 6.12 . [31, II. 4]. Let $S_{1}$ and $S_{2}$ be involutions on $X_{1}$ and $X_{2}$, with $\partial X_{1}=$ disjoint union $Y_{1} \cup X_{0}$ and $\partial X_{2}=$ disjoint union $Y_{2} \cup X_{0}$, and $S_{1}\left|X_{0}=S_{2}\right| X_{0}$. Let $(X, S)$ denote $\left(X_{1} \cup_{X_{0}} X_{2}, S_{1} \cup S_{2}\right)$. Then $\sigma_{s}(x)=$ $\sigma_{s_{1}}\left(X_{1}\right)+\sigma_{s_{2}}\left(X_{2}\right)$.

Now, we are ready to prove the folowing:

THEOREM 6.13. Let $\left(\Sigma^{2 n+1}, T\right)$ be a free involution on homotopy sphere $\Sigma^{2 n+1}$, where $n$ is odd and $\geqslant 3$, and $\left(\Sigma^{2 n+1}, T\right)=\left(\Sigma_{f}, T_{f}\right)$ for an A-diffeomorphism $f$ of $S^{n} \times S^{n}$. Then index $B_{M}^{f}=8 \sigma\left(\Sigma^{2 n+1}, T\right)$.

Proof. From the Mayer-Vietoris sequences, we see that $N=S^{n} \times D^{n} U_{\text {Id }}$ $M \cup_{f} D^{n+1} \times S^{n-1}$ is an $(n-1)$-connected characteristic submanifold of

$$
\left(\Sigma_{f}, T_{f}\right)=\left(S^{n} \times D^{n+1}, A\right) \cup_{\mathrm{Id}}\left(S^{n} \times S^{n} \times I, A \times \mathrm{Id}\right) \cup_{f}\left(D^{n+1} \times S^{n}, A\right) .
$$


$\Sigma_{f}=E \cup T_{f} E, E \cap T_{f} E=N$, and

$$
H_{n}(N)=\operatorname{Ker}\left(H_{n}(N) \rightarrow H_{n}(E)\right) \oplus \operatorname{Ker}\left(H_{n}(N) \rightarrow H_{n}\left(T_{f} E\right)\right),
$$

[8]. Let $K_{n}=\operatorname{Ker}\left(H_{n}(N) \rightarrow H_{n}(E)\right)$, and $B=$ the symmetric unimodular bilinear form defined on $K_{n}$ by $B(x \cdot y)=x \cdot T_{f *} y . \sigma\left(\Sigma_{f}, T_{f}\right)$ is defined to be $(1 / 8)$ index $B$.

Consider the sysmetric bilinear forms $C, C_{1}, C_{2}$, defined on

$$
H_{n}(N), H_{n}\left(S^{n} \times D^{n}\right), H_{n}\left(D^{n+1} \times S^{n-1}\right)
$$

respectively by $C(x, y)=x \cdot T_{f *} y, C_{1}(x, y)=x \cdot A_{*} y, C_{2}(x, y)=x \cdot A_{*} y$. Since $H_{n}\left(D^{n+1} \times S^{n-1}\right)=0$ and $H_{n}\left(S^{n} \times S^{n-1}\right) \rightarrow H_{n}\left(S^{n} \times D^{n}\right)$ is onto, index $C_{2}=$ index $C_{1}=0$. Hence index $C=\operatorname{index} C_{M}^{f}$ by (6.12). But index $C_{M}^{f}=2$ index $B_{M}^{f}$ by (6.11). Similarly index $C=2$ index $B[8, \mathrm{p} .75]$. Hence index $B_{M}^{f}=$ index $B=8 \sigma\left(\Sigma_{f}, T_{f}\right)=8 \sigma\left(\Sigma^{2 n+1}, T\right)$. Q.E.D.

REMARK 6.14. If the characteristic submanifold $M$ in (6.13) satisfies (6.8)(a), i.e. $H_{n-1}(M)=Q$, then the symmetric bilinear form $B_{M}^{f}$ defined on Ker $\alpha$ in (6.7) is actually isomorphic to the unimodular symmetric bilinear form $B$ defined on $K_{n}$ in (6.14). By the Mayer-Vietoris sequence we can show that if $j: \operatorname{Ker} \alpha \rightarrow K_{n}$ is an isomorphism under the map induced by the inclusion, then we show that for $x, y \in \operatorname{Ker} \alpha, x \cdot T_{*} y=j x \cdot j T_{*} y$, which follows from the fact that some multiples of $x$ and $T_{*} y$ can be represented by the immersions $h_{1}$ and $h_{2}$ of manifolds $M^{P}, N^{P} \rightarrow X^{2 P}$, [32], and Theorem V.1.3 of [5]: The geometric intersection number of $M$ and $N=$ the intersection number of the homology classes $h_{1 *}[M] \cdot h_{2 *}[N]$. Hence $B f_{M}$ is isomorphic to $B$. But given an involution $\left(\Sigma_{f}, T_{f}\right)$, we do not know whether we can always find such an $M$ or not.

Given a free involution on a homotopy sphere $\left(\Sigma^{2 n+1}, T\right), n \neq 3,7$, we can always find an $A$-diffeomorphism $f$ of $S^{n} \times S^{n}$, which is homotopic to identity, such that $\left(\Sigma^{2 n+1}, T\right)=\left(\Sigma_{f}, T_{f}\right)$ by (1.2). The next theorem tells us that the Browder-Livesay index invariant is additive: Given two involutions $\left(\Sigma_{f}, T_{f}\right)$ and $\left(\Sigma_{g}, T_{g}\right)$ with $f$ and $g$ homotopic to identity, we have $\sigma\left(\Sigma_{f}, T_{f}\right)+$ $\sigma\left(\Sigma_{g}, T_{g}\right)=\sigma\left(\Sigma_{g f}, T_{g f}\right)$.

THEOREM 6.15. If $f, g \in J_{2 n+1}=\varnothing^{-1}\left(D_{0}\left(S^{n} \times S^{n}\right)\right), n$ odd, then $\sigma\left(\Sigma_{f}, T_{f}\right)+\sigma\left(\Sigma_{g}, T_{g}\right)=\sigma\left(\Sigma_{g f}, T_{g f}\right)$.

Proof. Let $M, N^{\prime}$ be characteristic submanifolds for $(W, T)=\left(S^{n} \times S^{n} \times\right.$ $I, A \times$ Id) associated to $f, g$ respectively as in (6.6). $M$ and $N^{\prime}$ are (n-2)-connected, $H_{n-1}(M)$ and $H_{n-1}\left(N^{\prime}\right)$ are cyclic, $\partial M=f^{-1}\left(S^{n} \times S^{n-1}\right) \times 1-S^{n} \times$ $S^{n-1} \times 0$ and $\partial N^{\prime}=g^{-1}\left(S^{n} \times S^{n-1}\right) \times 1-S^{n} \times S^{n-1} \times 0$. Since $f^{-1} \times$ Id is an equivariant diffeomorphism for $(W, T), N=\left(f^{-1} \times \mathrm{Id}\right)\left(N^{\prime}\right)$ is a characteristic submanifold of $(W, T)$ and $\partial N=f^{-1} g^{-1}\left(S^{n} \times S^{n-1}\right) \times 1-f^{-1}\left(S^{n} \times S^{n-1}\right)$ 
$\times 0$. Furthermore, the bilinear form $C_{N^{\prime}}$ defined on $H_{n}\left(N^{\prime}\right)$ by $C_{N^{\prime}}(x, y)=x \cdot$ $T_{*} y$ is isomorphic to $C_{N}^{g}$, hence, index $C_{N^{\prime}}=\operatorname{index} C_{N}^{g}$.

Now, we glue two copies $W^{\prime}$ and $W^{\prime \prime}$ of $W$ together along $W_{1}$ of $W^{\prime}$ and $W_{0}$ of $W^{\prime \prime}$ by the identity. Consider $M \subseteq W^{\prime}$ and $N \subseteq W^{\prime \prime} . P=M \cup N$ is a characteristic submanifold for $\left(W^{\prime} \cup W^{\prime \prime}, T\right)=(W, T)$ with $\partial P=f^{-1} g^{-1}\left(S^{n} \times S^{n-1}\right) \times$ $1-S^{n} \times S^{n-1} \times 0$, and $P$ satisfies (6.6). $P$ is $(n-2)$-connected and $H_{n-1}(P)$ is cyclic by the Mayer-Vietoris sequences. Hence $C_{P}^{g f}$ and $B_{P}^{g f}$ are defined.

By (6.12) again, we have index $C_{P}^{g f}=\operatorname{index} C_{N^{\prime}}+\operatorname{index} C_{M}^{f}$. Hence index $C_{P}^{g f}=$ index $C_{N}^{g}+$ index $C_{M}^{f}$, which implies index $B_{P}^{g f}=$ index $B_{N}^{g}+$ index $B_{M}^{f}$ by (6.11). Thus $\sigma\left(\Sigma_{g f}, T_{g f}\right)=\sigma\left(\Sigma_{g}, T_{g}\right)+\sigma\left(\Sigma_{f}, T_{f}\right)$ by (6.13). Q.E.D.

REMARK 6.16. Actually, we have showed that given an $A$-diffeomorphism $f$ of $S^{n} \times S^{n}, n$ odd, we can associate an index $\beta(f)$ to $f$ which is defined to be the index of the form $B_{M}^{f}$ above. By the standard argument as in $[8,3.2]$, we see that $B_{M}^{f}$ is independent of the choice of the characteristic submanifold $M$. The proof of Theorem 6.15 shows that the induced map $\bar{\beta}: D\left(S^{n} \times S^{n}, A\right) \rightarrow Z$ is a homomorphism for $n \geqslant 3$.

7. The Arf invariant case. Theorem (6.15) is no longer valid in the case $n$ is even, as shown by the following example.

Proposition 7.1. If $n$ is even and $>2$, then there exist two A-diffeomorphisms $f, g$ of $S^{n} \times S^{n}$ such that $f$ and $g$ are homotopic to Id, and $\sigma\left(\Sigma_{f}, T_{f}\right)=$ $\sigma\left(\Sigma_{g}, T_{g}\right)=\sigma\left(\Sigma_{g f}, T_{g f}\right)=0$ but $\sigma\left(\Sigma_{f g}, T_{f g}\right)=1$.

Proof. Let $\tau$ be one of the generators of $\pi_{n}\left(S O_{n+1}\right)$. We choose a representative for $\tau$ such that $\tau(x)=\tau(-x)$, and define two $A$-diffeomorphisms $f$ and $g$ by $f(x, y)=(x, \tau(x) \cdot y), g(x, y)=(\tau(y) \cdot x, y)$. $f$ and $g$ are homotopic to Id, (4.4). It follows from (2.1) that $\left(\Sigma_{f}, T_{f}\right)=\left(\Sigma_{g}, T_{g}\right)=\left(\Sigma_{g f}, T_{g f}\right)=\left(S^{2 n+1}, A\right)$. Hence their Browder-Livesay invariant is 0 .

P. Orlik showed that if $\left(\Sigma^{4 k+1}, T\right)$ extends to an involution with fixed point on a $\pi$-manifold $W^{4 k+2}$ whose boundary is $\Sigma^{4 k+1}$, then $\sigma\left(\Sigma^{4 k+1}, T\right)=$ $C\left(W^{4 k+2}\right)$, the Arf invariant of $W^{4 k+2},[26],[21, \mathrm{p} .69]$. We will construct such a $W$ to show that $\sigma\left(\Sigma_{f g}, T_{f g}\right)=1$.

Following [22], we define $W$ to be

$$
\left(D^{n+1} \times D^{n+1}\right)_{1} \cup_{g}\left(D^{n+1} \times D^{n+1}\right)_{2} \cup_{f}\left(D^{n+1} \times D^{n+1}\right)_{3},
$$

where $g$ is the diffeomorphism gluing $\left(D^{n+1} \times S^{n}\right)_{1}$ and $\left(D^{n+1} \times S^{n}\right)_{2}$ together, $f$ is the gluing map from $\left(S^{n} \times D^{n+1}\right)_{2}$ to $\left(S^{n} \times D^{n+1}\right)_{3}$. Since $f$ and $g$ are $A$ equivariant, we define an involution $T^{\prime}$ on $W$ by gluing the antipodal map $A$ on each summand. The restriction of $T^{\prime}$ to $\partial W=\Sigma_{f g}$ is $T . C\left(W^{4 k+2}\right)=1$ follows from $[6, \mathrm{~V}]$. Q.E.D.

As in the index case, given an involution $\left(\Sigma^{2 n+1}, T\right)=\left(\Sigma_{f}, T_{f}\right)$ we can find an 
$(n-2)$-connected characteristic submanifold $M$ of $(W, T)=\left(S^{n} \times S^{n} \times I, A \times\right.$ Id $)$ with $\partial M=S^{n} \times S^{n-1} \times 0 \cup f^{-1}\left(S^{n} \times S^{n-1}\right) \times 1$ and $H_{n-1}(M)$ is cyclic.

In the rest of this section, all homology will be taken with $Z_{2}$ coefficients, unless stated otherwise explicitly.

As in (6.10), we have two cases: either (a) $H_{n-1}(M)=Z_{2}$, or (b) $H_{n-1}(M)$ $=0$. In case (a), since $Z=H_{n-1}\left(M_{i} ; Z\right) \rightarrow H_{n-1}(M ; Z)$ is onto (6.7), we see that $H_{n-1}\left(M_{1}\right) \rightarrow H_{n-1}(M)$ is an isomorphism.

Suppose $H_{n-1}(M)=Z_{2}$. We see that the map $i_{3}$ in (6.7) is trivial (taken with $Z_{2}$ coefficient). We define a bilinear form $B_{M}^{f}$ on $\operatorname{Ker} \alpha$ in (6.7) by $B_{M}^{f}=$ $x \cdot T_{*} y$. By using the Mayer-Vietoris sequences, and applying [32] and [5, V. 1.3] as in (6.14), we can show that $B_{M}^{f}$ is isomorphic to the bilinear form $B_{2}$ defined by Browder and Livesay in [8]. In particular, $B_{M}^{f}$ is unimodular.

Following [8], we can define a cohomology operation $\psi_{M}^{f}$ on $\operatorname{Ker} \alpha$, (for details, see $[8, \S 4])$. For $x, y \in \operatorname{Ker} \alpha$, we have $B_{M}^{f}(x, y)=\psi_{M}^{f}(x+y)+$ $\psi_{M}^{f}(x)+\psi_{M}^{f}(y),[8,4.5]$. Since $B_{M}^{f}$ is unimodular, the Arf invariant for $\psi_{M}^{f}$ is well-defined, [5], as follows. Choose a sympletic basis $x_{1}, \ldots, x_{n}, y_{1}, \ldots, y_{n}$ for $\operatorname{Ker} \alpha$ such that $B_{M}^{f}\left(x_{i}, y_{j}\right)=\delta_{i j}, B_{M}^{f}\left(x_{i}, x_{j}\right)=B_{M}^{f}\left(y_{i}, y_{j}\right)=0$, the Arf invariant $c_{1}(f, M)=\sum_{i=1}^{n} \psi_{M}^{f}\left(x_{i}\right) \psi_{M}^{f}\left(y_{i}\right)$.

LEMMA 7.2. Suppose $H_{n-1}(M)=Z_{2}$ in (6.7), and $\psi_{M}^{f}$ is defined as in [8]. Then any $x \in \operatorname{Ker} \alpha$ can be represented by an immersed manifold $X^{n}$, and $\psi_{M}^{f}(x)=1$ iff $X \cap T X$ in general position consists of an odd number of pairs of points.

Proof. The representability of $x \in \operatorname{Ker} \alpha$ by an immersed manifold follows from [32], since the coefficient group is $Z_{2}$. The other assertion can be proved by exactly the same argument in $[8,4.6]$. Q.E.D.

Proposition 7.3. For $n$ even, if the manifold $M$ associated to $\left(\Sigma_{f}, T_{f}\right)$ as in (6.7) with $Z_{2}$-coefficient satisfies $H_{n-1}(M)=Z_{2}$, then $c_{1}(f, M)=\sigma\left(\Sigma_{f}, T_{f}\right)$.

Proof. We noted before that $B_{M}^{f}$ is isomorphic to $B_{2}$ in this case. Let $\psi$ be the quadratic form associated to $B_{2}$ defined in [8]. From $(7.2),[8,4.6]$, and $\left[5\right.$, V. 1.3] again, we see that $\psi_{M}$ and $\psi$ are isomorphic. Hence their Arf invariants are equal. Q.E.D.

Proposition 7.4. For $n$ even, and $f, g \in J_{2 n+1}$. If there exist $M, N$ associated to $\left(\Sigma_{f}, T_{f}\right),\left(\Sigma_{g}, T_{g}\right)$ as in (6.6) such that $H_{n-1}(M)=H_{n-1}(N)=Z_{2}$, then $\sigma\left(\Sigma_{f}, T_{f}\right)+\sigma\left(\Sigma_{g}, T_{g}\right)=\sigma\left(\Sigma_{g f}, T_{g f}\right)$.

Proof. As in (6.15), let $P$ denote $M \cup N$, the characteristic submanifold associated to $\left(\Sigma_{g f}, T_{g f}\right)$. We denote the domain on which $B_{M}^{f}, B_{N}^{g}, B_{P}^{g f}$ is defined by $\operatorname{Ker} \alpha, \operatorname{Ker} \beta, \operatorname{Ker} \gamma$ respectively. By the Mayer-Vietoris sequence, we see that $H_{n-1}(P)=Z_{2}$, and $\operatorname{Ker} \gamma=\operatorname{Ker} \alpha \oplus \operatorname{Ker} \beta$ under the inclusion. Using (7.2) and 
$\left[5\right.$, V. 1.3] as in (7.3), we see that the quadratic form $\psi_{P}^{g f}$ is the direct sum of $\psi_{M}^{f}$ and $\psi_{N}^{g}$. Hence $\sigma\left(\Sigma_{f}, T_{f}\right)+\sigma\left(\Sigma_{g f}, T_{g f}\right)$ by (7.3). Q.E.D.

CoROllary 7.5. Let $f$ be the A-diffeomorphism defined in (7.1). $M$ is an $(n-2)$-connected characteristic submanifold for $\left(S^{n} \times S^{n} \times I, A \times I d\right)$ such that $\partial M=f^{-1}\left(S^{n} \times S^{n-1}\right) \times 1-S^{n} \times S^{n-1} \times 0$. If $H_{n-1}(M)$ is cyclic, then $H_{n-1}\left(M ; Z_{2}\right)=0$.

Proof. Let $g$ be the $A$-diffeomorphism defined in (7.1) by $g(x, y)=$ $(\tau(y) \cdot x, y)$. Since $g\left(S^{n} \times S^{n-1}\right)=S^{n} \times S^{n-1}$, we can take $N=S^{n} \times S^{n-1}$ $\times I$ to be a characteristic manifold associated to $\left(\Sigma_{g}, T_{g}\right)$ as in (6.6). $H_{n-1}\left(N ; Z_{2}\right)$ $=Z_{2}$. Assume $H_{n-1}\left(M ; Z_{2}\right)=Z_{2}$. From (7.4), we would have $\sigma\left(\Sigma_{f g}, T_{f g}\right)=$ $\sigma\left(\Sigma_{f}, T_{f}\right)+\sigma\left(\Sigma_{g}, T_{g}\right)=0$. This contradicts (7.1). Hence $H_{n-1}\left(M ; Z_{2}\right)=0$. Q.E.D.

8. Curious involutions. Let $\Sigma_{0}^{4 k-1}$ denote the generator of $b P^{4 k}$, a cyclic subgroup of $\Gamma^{4 k-1}$, consisting of those homotopy spheres which bound parallelizable manifolds, [17]. Let $\left(\Sigma^{4 k-1}, T\right)$ be a fixed point free involution such that $\Sigma^{4 k-1} \in b P_{4 k}$, we can write $\Sigma^{4 k-1}=m \Sigma_{0}^{4 k-1}$ for some integer $m$, which is well-defined mod 2. Following [13], we will call an involution $\left(\Sigma^{4 k-1}, T\right)$ curious if $m+\sigma\left(\Sigma^{4 k-1}, T\right) \bmod 2$ is equal to 1 .

LEMMA 8.1. The number of curious involutions $\left(\Sigma_{0}^{4 k-1}, T\right)$ with $\sigma\left(\Sigma_{0}^{4 k-1}, T\right)$ $=0$ is finite.

Proof. The number of the normal cobordism classes $\left[P^{4 k-1}, G / O\right]$ is finite, [21]. In each normal cobordism class, there is exactly one p.l. involution with the zero Browder-Livesay's index invariant, [21] or [33]. Since $\pi_{j}(P L / O)$ is finite, the number of differentiable involutions with zero index invariant in each normal cobordism class is finite by smoothing theory. Q.E.D.

Let $S^{n}$ denote the standard sphere. As an application of our previous theorems, we have the following:

THEOREM 8.2. Let $n=8 k+3$; the number of curious involutions $\left(\Sigma_{0}^{n}, T\right)$ with $\sigma\left(\Sigma_{0}^{n}, T\right)=0$ is either 0 or equal to the number of involutions $\left(S^{n}, T\right)$ with $o\left(S^{n}, T\right)=0$.

Proof. From (5.2), we know that $h S\left(P^{8 k+3}\right)$ is in $1-1$ correspondence with $J_{8 k+3}=\varnothing^{-1}\left(D_{0}\left(S^{4 k+1} \times S^{4 k+1}\right)\right)$, a subgroup of the group

$$
D_{0}\left(S^{4 k+1} \times S^{4 k+1}, A\right)
$$

of concordance classes of $A$-diffeomorphisms of $S^{4 k+1} \times S^{4 k+1}$, consisting of those $A$-diffeomorphisms which are homotopic to identity.

Let $C=\left\{f \in J_{8 k+3} \mid \Sigma_{f}=\Sigma_{0}^{8 k+3}, \sigma\left(\Sigma_{f}, T_{f}\right)=0\right\}$, and $C^{\prime}=\left\{f \in J_{8 k+3} \mid \Sigma_{f}\right.$ $\left.=S^{8 k+3}, \sigma\left(\Sigma_{f}, T_{f}\right)=0\right\}$. There are two cases: 
(a) $k$ is odd. $D_{0}\left(S^{4 k+1} \times S^{4 k+1}\right)=\Gamma^{8 k+3}$ by (5.2). Let $\gamma \in \Gamma^{8 k+3}$ be the element which corresponds to $\Sigma_{0}^{8 k+3}$. If $C$ is not empty, then $\varnothing(f)=\gamma$ for all $f \in C$, where $\varnothing: D_{0}\left(S^{4 k+1} \times S^{4 k+1}, A\right) \rightarrow D_{0}\left(S^{4 k+1} \times S^{4 k+1}\right)$ is the forgetting map in $\S 4$. Take $g \in C, \varnothing\left(g^{-1}\right)=\gamma^{-1}$, hence $\Sigma_{g^{-1}}=-\Sigma_{0}^{4 k+3}$; and $\sigma\left(\Sigma_{g^{-1}}, T_{g^{-1}}\right)=-\sigma\left(\Sigma_{g}, T_{g}\right)=0$ by (6.15). Using (6.15) again, we see that the mapping $f \rightarrow f \circ g^{-1}$ for $f \in C$ maps $C$ into $C^{\prime}$ because $\Sigma_{f g-1}=S^{4 k+3}$. This correspondence is $1-1$ and onto, since the inverse is given by $h \rightarrow h \circ g$ for $h \in C^{\prime}$.

(b) $k$ is even. From (5.2), we know that $D_{0}\left(S^{4 k+1} \times S^{4 k+1}\right)$ is the semidirect product of $\left(\sigma_{1}\right)+\Gamma^{8 k+3}$ and $\left(\sigma_{2}\right)$, where $\sigma_{1}$ and $\sigma_{2}$ are defined by $\sigma_{1}(x, y)=(x, \sigma(x) \cdot y)$ and $\sigma_{2}(x, y)=(\sigma(y) \cdot x, y), \sigma_{1}^{2}=\sigma_{2}^{2}=\mathrm{Id}$ in $D_{0}\left(S^{4 k+1} \times S^{4 k+1}\right)$, [19]. If $C$ is not empty, then for $f \in C, \varnothing(f)=\gamma, \sigma_{2} \gamma \sigma_{1}$, $\gamma \sigma_{1}$, or $\sigma_{2} \gamma,[19]$, where $\gamma$ is the element of $\Gamma^{8 k+3}$ corresponding to $\Sigma_{0}^{8 k+3}$.

(i) If there exists a $g \in C$ such that $\varnothing(g)=\gamma$. Since $\gamma^{-1}$ lies in the center of $D_{0}\left(S^{4 k+1} \times S^{4 k+1}\right),[19]$, and $\varnothing\left(g^{-1}\right)=\gamma^{-1}$, we see that $\varnothing\left(g f^{-1}\right)=$ Id, $\sigma_{2} \sigma_{1}, \sigma_{1}$, or $\sigma_{2}$. Hence $\Sigma_{f g-1}=S^{8 k+3}$. By applying (6.15) as in. (a), we see that the mapping $f \rightarrow f \circ g^{-1}$ for $f \in C$ gives a $1-1$ correspondence between $C$ and $C^{\prime}$.

(ii) If $\varnothing(f) \neq \gamma$ for every $f \in C$, but there exists $g \in C$ such that $\varnothing(g)=$ $\gamma \sigma_{1}$. Then $\varnothing\left(g^{-1}\right)=\sigma_{1}^{-1} \gamma^{-1}=\sigma_{1} \gamma^{-1}$, and $\sigma\left(\Sigma_{g^{-1}}, T_{g^{-1}}\right)=-\sigma\left(\Sigma_{g}, T_{g}\right)=0$ by (6.15). In this case, $\varnothing(f)=\gamma \sigma_{1}, \sigma_{2} \gamma$, or $\sigma_{2} \gamma \sigma_{1}$ for $f \in C$. As in (i), we have $\varnothing\left(f g^{-1}\right)=$ Id, $\sigma_{2} \sigma_{1}$, or $\sigma_{2}$. Hence $\Sigma_{f g-1}=S^{8 k+3}$. By (6.15) again, the mapping $f \rightarrow f \circ g^{-1}$ for $f \in C$ gives a $1-1^{f g}$ correspondence between $C$ and $C^{\prime}$.

(iii) If $\varnothing(f) \neq \gamma, \gamma \sigma_{1}$, for every $f \in C$, but there exists $g \in C$ such that $\varnothing(g)=\sigma_{2} \gamma$. Then the mapping $f \rightarrow g^{-1} f$ gives a $1-1$ correspondence between $C$ and $C^{\prime}$ as in (i).

(iv) If $\varnothing(f)=\sigma_{2} \gamma \sigma_{1}$ for all $f \in C$. Take $g \in C, \varnothing\left(g^{-1}\right)=\sigma_{1}^{-1} \gamma^{-1} \sigma_{2}^{-1}$, and $\sigma\left(\Sigma_{g^{-1}}, T_{g^{-1}}\right)=0$ by (6.15) as before. For $f \in C, f \rightarrow f \circ g^{-1}$ gives $1-1$ correspondence between $C$ and $C^{\prime}$ by (6.15) as before. Q.E.D.

9. Decomposition of $\left(\Sigma^{2 n}, T\right)$. In this section, we will prove an analogue of (1.1) for free involutions on even dimensional homotopy spheres.

Proposition 9.1. For $n>3,\left(\Sigma^{2 n}, T\right)=\left(S^{n} \times D^{n}, A\right) \cup_{g}\left(D^{n+1} \times\right.$ $\left.S^{n-1}, A\right)$ for some A-diffeomorphism $g$ of $\left(S^{n} \times S^{n-1}, A\right)$.

Proof. Let $P^{m}$ denote the real projective space. There is a homotopy equivalence $f: P^{2 n} \rightarrow Q^{2 n}=\Sigma^{2 n} / T$. Let $i: P^{n} \rightarrow P^{2 n}$ be the inclusion. For dimensional reasons, $f \mid P^{n}$ is homotopic to an embedding by [12]. By the homotopy extension theorem, we see that $f$ homotopic to a smooth map $g$ such that $g \mid P^{n}$ is an embedding.

Let $\nu_{1}$ denote the normal bundle of $P^{n}$ in $P^{2 n}$, and $\nu_{2}$ the normal bundle of $g P^{n}$ in $Q^{2 n}$. Let $\tau_{1}$ and $\tau_{2}$ denote the tangent bundles of $P^{2 n}$ and $Q^{2 n}$. By 
Theorem 3.6 in [1], $g^{*} \tau_{2}$ and $\tau_{1}$ are $J$-equivalent. Since the projection $\widetilde{K O}\left(P^{2 n}\right)$ $\rightarrow J\left(P^{2 n}\right)$ is an isomorphism, $g^{*} \tau_{2}$ and $\tau_{1}$ are stably equivalent. Let $\tau_{p}$ denote the tangent bundle of $P^{n} \cdot g^{*} \tau_{2} \mid P^{n}$ is stably equivalent to $\tau_{1} \mid P^{n}$. Since $g \mid P^{n}$ is an embedding, $\left(g \mid P^{n}\right)^{*}\left(\tau_{1} \mid g P^{n}\right)$ is stably equivalent to $\tau_{1} \mid P^{n}$. The induced map commutes with the Whitney sum; hence $\tau_{p} \oplus \nu_{1}$ is stably equivalent to $\tau_{p} \oplus$ $g^{*} \nu_{2}$. By adding a stable inverse for $\tau_{p}$, we see that $\nu_{1}$ is stably equivalent to $g^{*} \nu_{2}$. But $\nu_{1}$, the normal bundle of $P^{n}$ in $P^{2 n}$, is equivalent to $n \eta=\eta \oplus \eta \oplus$ - $\oplus \eta, n$ times, where $\eta$ is the canonical line bundle over $P^{n}$. Hence by Corollary 1.10 in [5], $g^{*} \nu_{2}$ and $\nu_{1}$ are actually equivalent. By lifting this equivalence of normal bundles to the double cover, we see that there is an equivariant embedding $h=\left(S^{n} \times D^{n}, A\right) \rightarrow\left(\Sigma^{2 n}, T\right)$. The image solid torus is unknotted by [12], the complement is diffeomorphic to $D^{n+1} \times S^{n-1}$ by the $h$-cobordism theorem.

Consider $\left(S^{n} \times D^{n}, A\right) \stackrel{h}{\rightarrow}\left(\Sigma^{2 n}, T\right) \stackrel{k^{\prime}}{\longleftarrow}\left(D^{n+1} \times S^{n-1}, U\right)$, where we define an involution on the right-hand torus by $U=k^{\prime-1} T k^{\prime}$. Both $h$ and $k^{\prime}$ are equivariant embeddings. The $A$-invariant diagonal sphere in $S^{n-1} \times S^{n-1} \subseteq$ $S^{n} \times S^{n-1}$ on the left-hand side is mapped by $k^{\prime} h \mid S^{n} \times S^{n}$ onto a $U$-invariant sphere $S_{\Delta}^{n-1}$ on the right. On the boundary of $D^{n+1} \times S^{n-1}, U$ is equivalent to $A$. We equivariantly collar $S^{n} \times S^{n-1}$ in $\left(D^{n+1} \times S^{n-1}, U\right)$ by $[9,21.2]$, and push $S_{\Delta}^{n-1}$ a little way inside the boundary. $U$ is equivalent to $A$ on a tubular neighborhood $N$ of this interior copy of $S_{\Delta}^{n-1}$, which can be proved by applying Lemma 2 of [20] to show that the normal bundle of $P^{n-1}=S_{\Delta}^{n-1} / A$ in $N$ is equivalent to the normal bundle of $P^{n-1}$ in $P^{2 n}$. The orbit space $\left(D^{n-1} \times S^{n-1}-\stackrel{\circ}{N}\right) / U$ is an $h$-cobordism between two copies of a manifold diffeomorphic to $S^{n} \times S^{n-1} / A$. Since the Whitehead group $W h\left(Z_{2}\right)=0$, this $h$-corbodism is diffeomorphic to $\left(S^{n} \times S^{n-1} / A\right) \times I$ by the $s$-cobordism theorem. Therefore $\left(D^{n+1} \times S^{n-1}, U\right)$ is equivalent to $\left(D^{n+1} \times S^{n-1}, A\right)$. Q.E.D.

Similar to (2.1), we have the following:

PROPOSITION 9.2. $n>3,\left(\Sigma^{2 n}, T\right)=\left(\Sigma_{f}, T_{f}\right)=\left(\Sigma_{g}, T_{g}\right)$ for some A-diffeomorphisms $f$ and $g$ of $S^{n} \times S^{n-1}$ iff there exists A-diffeomorphisms $H: S^{n} \times$ $D^{n} \rightarrow S^{n} \times D^{n}$ and $K: D^{n+1} \times S^{n-1} \rightarrow D^{n+1} \times S^{n-1}$ such that $f=K g H$ on $S^{n} \times S^{n-1}$.

ProOF. Exactly the same as in [20]. Suppose we have two distinct decompositions, $\left(S^{n} \times D^{n}, A\right) \stackrel{h_{i}}{\longrightarrow}\left(\Sigma^{2 n}, T\right) \stackrel{k_{i}}{\longleftarrow}\left(D^{n+1} \times S^{n-1}, A\right), i=1$ or 2 . On $S^{n} \times S^{n-1}, f=k_{1}^{-1} h_{1}$ and $g=k_{2}^{-1} h_{1}$. Note that $h_{1}, h_{2}=S^{n} \times 0 \rightarrow \Sigma^{2 n+1}$ are equivariantly homotopic embeddings, since both are lifted classifying maps for $P^{n}$ in $Q^{2 n}$. Hence $h_{1}\left(S^{n} \times 0\right)$ and $h_{2}\left(S^{n} \times 0\right)$ are equivariantly isotopic by a global isotopy by [12] as in the proof of (2.4). By the equivariant tubular neighborhood theorem, [3], there is an equivariant isotopy of $\Sigma^{2 n}$ such that, after composing with the first isotopy, there is an equivariant diffeomorphism $r: \Sigma^{2 n} \rightarrow$ 
$\Sigma^{2 n}$ with $H=h_{2}^{-1} r h_{1}$, an equivariant bundle map. Let $K=k_{1}^{-1} r^{-1} k_{2}: D^{n+1} \times$ $S^{n-1}$; we note that $k_{1}=r^{-1} k_{2} K^{-1}$. Hence $f=k_{1}^{-1} h_{1}=K k_{2}^{-1} r h_{1}=K k_{2}^{-1} h_{2} H$ $=K g H$. $H$ extends equivariantly to all of $S^{n} \times D^{n}$, and $H$ extends equivariantly to all of $D^{n+1} \times S^{n-1}$. Q.E.D.

10. Equivariant Milnor's pairing. Milnor defined in [22] a certain pairing $\Sigma$ on $s_{*} \pi_{n}\left(S O_{n}\right) \otimes \pi_{n}\left(S O_{n+1}\right)$ to $\Gamma^{2 n+1}$, the group of homotopy spheres, where $s_{*} \pi_{n}\left(S O_{n}\right)$ denotes the image of $s_{*}: \pi_{n}\left(S O_{n}\right) \rightarrow \pi_{n}\left(S O_{n+1}\right)$. The pairing is defined as follows. Let $a \in \pi_{n}\left(S O_{n}\right), a^{\prime} \in \pi_{n}\left(S O_{n+1}\right)$; define two diffeomorphisms $f_{1}$ and $f_{2}$ on $S^{n} \times S^{n}$ by $f_{1}(x, y)=\left(x, s_{*} a(x) \cdot y\right)$ and $f_{2}(x, y)=\left(a^{\prime}(y) \cdot x, y\right)$. Let $h=f_{1} \circ f_{2}, \Sigma\left(s_{*} a, a^{\prime}\right)=S^{n} \times D^{n+1} \cup_{h} D^{n-1} \times S^{n}$.

If the above $s_{*} a$ and $a^{\prime}$ lie in the image of $\pi^{*}:\left[P^{n}, S O_{n+1}\right] \rightarrow \pi_{n}\left(S O_{n+1}\right)$, then we can take representatives of $s_{*} a$ and $a^{\prime}$ such that $s_{*} a(x)=s_{*} a(-x)$ and $a^{\prime}(x)=a^{\prime}(-x)$. Hence $h=f_{1} \circ f_{2}$ is an $A$-diffeomorphism, and $\Sigma\left(s_{*} a, a^{\prime}\right)$ admits a free involution, which is $\left(\Sigma_{h}, T_{h}\right)$. In $\S 7$, we used $a^{\prime}=s_{*} a=\tau$ in $\pi_{n}\left(S O_{n+1}\right), n$ even, to construct an involution on the Kervaire sphere with nonzero Arf invariant. For $n$ odd, we will see that all the involutions obtained in this way have zero index invariant.

From (3.1) and (3.9), we know that in $\pi_{n}\left(S O_{n+1}\right), s_{*} \pi_{n}\left(S O_{n}\right) \cap \pi^{*}\left[P^{n}, S O_{n+1}\right]$ $=\{2 \sigma\}$ for $n>7, n \equiv 3 \bmod 4$; and $=0$ for $n \equiv 1 \bmod 4$. Hence we only have to consider the case where $n \equiv 3 \bmod 4$ and $n \neq 3,7$.

Lemma 10.1. If $f$ is an A-diffeomorphism of $S^{n} \times S^{n}$ such that $f$ leaves $S^{n} \times S^{n-1}$ or $S^{n-1} \times S^{n}$ invariant, then $\sigma\left(\Sigma_{f}, T_{f}\right)=0$.

Proof. $S^{n} \times D^{n} \cup_{f} D^{n+1} \times S^{n-1}$ or $S^{n-1} \times D^{n+1} \cup_{f} D^{n} \times S^{n}$ is a codim 1 invariant sphere of $\left(\Sigma_{f}, T_{f}\right)$, because $S^{n} \times S^{n-1}, S^{n} \times D^{n}, D^{n+1} \times$ $S^{n-1}$, etc. are all invariant under $A$. Q.E.D.

In $\pi_{n}\left(S O_{n+1}\right)$ for $n \equiv 3 \bmod 4$ and $n \neq 3,7, \pi^{*}\left[P^{n}, S O_{n+1}\right]=Z+Z$, generated by $\tau$ and $2 \sigma ; \pi^{*}\left[P^{n}, S O_{n+1}\right] \cap s_{*} \pi_{n}\left(S O_{n}\right)=Z$, generated by $2 \sigma$, (3.9).

We first consider $\Sigma(2 \sigma, 2 \sigma)$. Let $\sigma=s_{*} a$, where $a \in \pi_{n}\left(S O_{n}\right),[18]$. We can choose a representative for $2 a$ such that $2 a(x)=2 a(-x)$. Let $f$ represent $a \in \pi_{n}\left(S O_{n}\right)$ such that $f \mid$ the southern hemisphere = identity. Since $n$ is odd, $A f$ is homotopic to $f$. The map $g: S^{n} \rightarrow S O_{n}$ defined by $g=f$ on the northern hemisphere and $=A f$ on the southern hemisphere represents $f+A f$, hence $2 a$, and $g(x)=g(-x)$. We have $f_{1}(x, y)=\left(x, s_{*} 2 a(x) \cdot y\right), f_{2}(x, y)=\left(s_{*} 2 a(y) \cdot x\right.$, $y$ ), and $h=f_{1} \circ f_{2}$. Since $2 a \in \pi_{n}\left(S O_{n}\right), s_{*} a\left(S^{n}\right) \cdot S^{n-1} \subseteq S^{n-1}, f_{1}\left(S^{n} \times S^{n-1}\right)$ $\subseteq S^{n} \times S^{n-1}$. But $2 a(x) \in S O_{n}$, which has a matrix representation [2a(x)]. We define $d \in \pi_{n}\left(S O_{n}\right)$ by $d(x)=[2 a(x)]^{-1}$, the inverse matrix for $[2 a(x)]$. The diffeomorphism $f_{3}$ defined by $f_{3}(x, y)=\left(x, s_{*} d(x) \cdot y\right)$ is the inverse for $f_{1}$, and $f_{3}\left(S^{n} \times S^{n-1}\right) \subseteq S^{n} \times S^{n-1}$. Hence $f_{1}\left(S^{n} \times S^{n-1}\right)=S^{n} \times S^{n-1}$. Similarly, 
$f_{2}\left(S^{n} \times S^{n-1}\right)=S^{n} \times S^{n-1}$. Thus $h=f_{1} \circ f_{2}$ leaves $S^{n} \times S^{n-1}$ invariant. $\sigma\left(\Sigma_{h}, T_{h}\right)=0$ by $(10.1)$.

Now, we consider the $A$-diffeomorphisms $g_{1}$ and $g_{2}$ of $S^{n} \times S^{n}$ defined by $g_{2}=f_{2}$ in the preceding paragraph and $g_{1}(x, y)=(\tau(y) \cdot x, y)$, where $\tau(y)=$ $\alpha(y) \alpha(e)$ as in $\S 3$. $e$ is the north pole of $S^{n}, \alpha(y)=$ the reflection through the hyperplane orthogonal to $y$. This representative of $\tau$ satisfies $\tau(x)=\tau(-x)$. $\tau\left(S^{n-1}\right) \cdot S^{n}=\alpha\left(S^{n-1}\right) \alpha(e) S^{n}=\alpha\left(S^{n-1}\right) S^{n}=S^{n}$. Hence $g_{1}\left(S^{n} \times S^{n-1}\right) \subseteq$ $S^{n} \times S^{n-1}$. But $\alpha(y) \alpha(y)=$ identity. Thus $g_{1}\left(S^{n} \times S^{n-1}\right)=S^{n} \times S^{n-1} \cdot h=$ $g_{2} \circ g_{1}$ leaves $S^{n} \times S^{n-1}$ invariant. $\sigma\left(\Sigma_{n}, T_{n}\right)=0$ by $(10.1)$.

Suppose $\beta_{1}$ and $\beta_{2}$ are two representatives for $\beta \in \pi_{n}\left(S O_{n+1}\right)$ such that $\beta_{i}(x)=\beta_{i}(-x)$; we define two $A$-diffeomorphisms $h_{1}$ and $h_{2}$ by $h_{i}(x, y)=(x$, $\left.\beta_{i}(x) \cdot y\right)$. Since $\pi^{*}\left[P^{n}, S O_{n+1}\right] \rightarrow \pi_{n}\left(S O_{n+1}\right)$ is $1-1$ for $n \equiv 3 \bmod 4$ and $n>7$, (3.9), we see that $h_{1}$ and $h_{2}$ are $A$-concordant by (2.4) and (2.5). Hence the construction of the $\left(\Sigma_{h}, T_{h}\right)$ is independent of the choice of representatives for $\beta \in$ Image $\pi^{*}$. Thus we have

Proposition 10.2. Every involution $\left(\Sigma^{8 n+7}, T\right)$ constructed above by using Milnor's pairing: $\Sigma=\Sigma(2 m \sigma, 2 n \sigma)$ or $\Sigma(2 m \sigma, n \tau)$, has zero Browder-Livesay index invariant.

Added in proof. Lemma 6.11 is not true. Since $T$ changes the orientation of the characteristic submanifold $M$, we have

$$
\text { index } C_{M}^{f} \mid \operatorname{Ker} \alpha=- \text { index } C_{M}^{f} \mid \operatorname{Ker} \alpha^{\prime} .
$$

Thus index $C_{M}^{f}=0$.

Here we will adapt the proof of [31, Theorem II.4] (instead of applying the Theorem itself, which was stated as Theorem 6.12 above) to verify Theorems 6.13 and 6.15 .

Let $M, N$, and $P$ be the characteristic submanifolds for $(W, T)=\left(S^{n} \times S^{n} \times\right.$ $I, A \times$ id) associated with $f, g$, and $g f$ respectively as in (6.15)

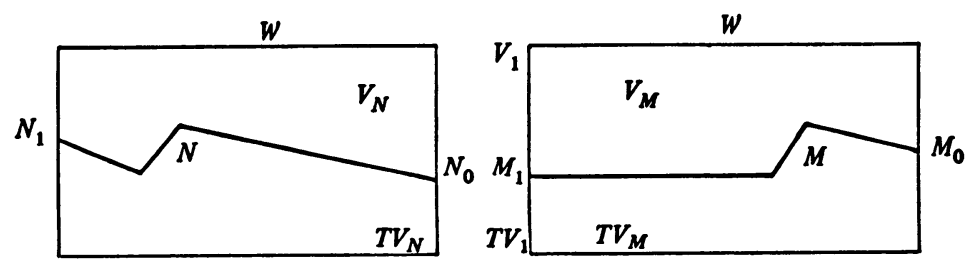

$$
\begin{aligned}
& M_{0}=S^{n} \times S^{n-1}, M_{1}=f^{-1}\left(S^{n} \times S^{n-1}\right)=N_{0}, \quad N_{1}=f^{-1} g^{-1}\left(S^{n} \times S^{n-1}\right), \\
& V_{1}=f^{-1}\left(S^{n} \times D^{n}\right), \quad P=M \cup_{M_{1}} N, V=V_{M} \cup_{V_{1}} V_{N}, \text { etc. }
\end{aligned}
$$

Let 


$$
\begin{aligned}
& \left(\alpha_{1}, \alpha_{1}^{\prime}\right): H_{n} M \rightarrow H_{n} V_{M} \oplus H_{n} T V_{M}, \\
& \left(\alpha_{2}, \alpha_{2}^{\prime}\right): H_{n} N \rightarrow H_{n} V_{N} \oplus H_{n} T V_{N}, \text { and } \\
& \left(\alpha, \alpha^{\prime}\right): H_{n} P \rightarrow H_{n} V \oplus H_{n} T V
\end{aligned}
$$

be the maps induced by inclusion as in (6.7).

THEOREM .

$$
\text { index } B_{P}^{g f}=\operatorname{index} B_{N}^{g}+\operatorname{index} B_{M}^{f} \text {. }
$$

Proof. (6.10) and (6.11) stated that

$$
H_{n} M=\operatorname{Ker} \alpha_{1} \oplus \operatorname{Ker} \alpha_{1}^{\prime} \oplus \operatorname{Im} i_{1},
$$

where $i_{1}: H_{n} \partial M \rightarrow H_{n} M$ is the inclusion. We take $Q$ as coefficient from now on. Let $m=$ rank $H_{n}\left(V, V_{1}\right)$. We can classify $M$ into two types:

(a) $H_{n-1} M=Q$, rank $H_{n} M=2 m+1$, rank $\operatorname{Ker} \alpha_{1}=m$, and $Q=H_{n-1} M_{1}$ $\rightarrow H_{n-1} M$ is onto.

(b) $H_{n-1} M=0$, rank $H_{n} M=2 m$, rank Ker $\alpha_{1}=m-1$.

Consider the following exact sequence.

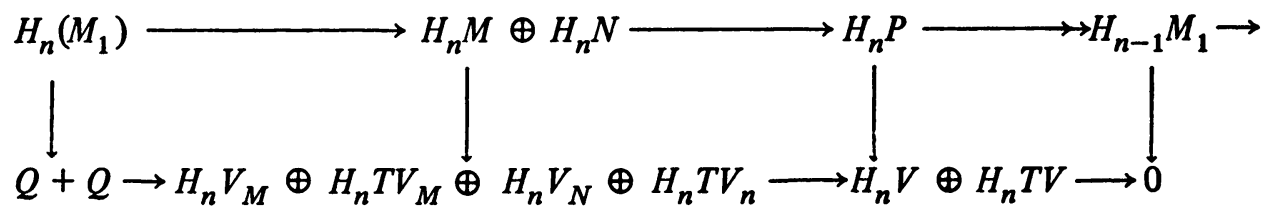

If one of $M, N$ is of type (a), then we can show that $\operatorname{Ker} \alpha_{1}+\operatorname{Ker} \alpha_{2} \rightarrow \operatorname{Ker} \alpha$ is an isomorphism by a simple counting argument, and index $B_{P}^{g f}=\operatorname{index} B_{N}^{g}+$ index $B_{M}^{f}$ follows from the statement in (6.14).

Now we assume that both $M$ and $N$ are of type (b). Let $\left(\alpha^{*}, \alpha^{\prime *}\right): H^{n} V$ $\oplus H^{n} T V \rightarrow H^{n} P$ be the map induced by inclusions. We have the following exact sequence.

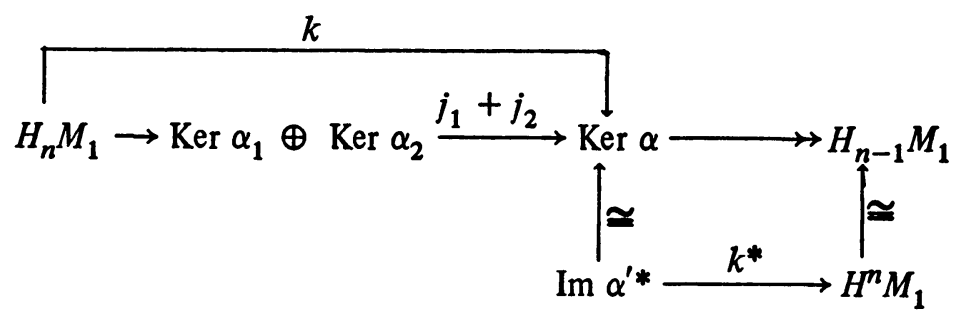

Let $A_{B} \operatorname{Im} k$ denote the annihilator of $\operatorname{Im} k$ under $B_{P}^{g f}$. Then we may use the argument in [31. II4] to show that $A_{B} \operatorname{Im} k=\operatorname{Im} j_{1}+\operatorname{Im} j_{2}$. But we also have

LeMma [31, II.3]. If $B$ is a symmetric bilinear form on a vector space $V$, and if there is a subspace $C \subseteq V$ with $C \subseteq A C$, then index $B=\operatorname{index} B \mid A C$. 
Thus our theorem follows from this Lemma and (6.14) as above. Q.E.D. The proof of Theorem 6.13 is similar.

\section{BIBLIOGRAPHY} \#6626.

1. J. F. Adams, On the groups $J(X)$. II, Topology 3 (1965), 137-171. MR 33

2. I. Berstein, Involutions with nonzero Arf invariant, Bull. Amer. Math. Soc. 74 (1968), 678-682. MR 38 \#5225.

3. G. E. Bredon, Introduction to compact transformation groups, Academic Press, New York, 1972.

4. W. Browder, Structures on $M \times R$, Proc. Cambridge Philos. Soc. 61 (1965), 337-

345. MR $30 \# 5321$.

5. - Surgery on simply-connected manifolds, Ergebnisse der Mathematik und ihrer Grenzgebiete, Band 65, Springer-Verlag, Berlin and New York, 1972.

6. - Cobordism invariants, the Kervaire invariant and fixed point free involutions, Trans. Amer Math. Soc. 178 (1973), 193-225. MR 48 \#3067.

7. W. Browder and G. R. Livesay, Fixed point free involutions on homotopy spheres, Bull. Amer. Math. Soc. 73 (1967), 242-245. MR 34 \#6781.

8. - Fixed point free involutions on homotopy spheres, Tôhoku Math. J. (2) 25 (1973), 69-87. MR 47 \#9610.

9. P. E. Conner and E. E. Floyd, Differentiable periodic maps, Ergebnisse der Mathematik und ihrer Grenzgebiete, N. F., Band 33, Springer-Verlag, Berlin; Academic Press, New York, 1964. MR 31 \#750.

10. A. Dold, Über fasernweise homotopieäquivalenz von Faserräumen, Math. Z. 62 (1955), 111-136. MR 17, 519.

11. M. Fujii, KO-groups of projective spaces, Osaka J. Math. 4 (1967), 141-149. MR 36 \#2143.

12. A. Haefliger, Plongements différentiable de variétés dans variétés, Comment. Math. Helv. 36 (1961), 47-82. MR 26 \#3069.

13. M. W. Hirsch and J. Milnor, Some curious involutions of spheres, Bull. Amer. Math. Soc. 70 (1964), 372-377. MR 31 \#751.

14. D. Husemoller, Fibre bundles, McGraw-Hill, New York, 1966. MR 37 \#821.

15. I. James and E. Thomas, An approach to the enumeration problem for non-stable vector bundles, J. Math. Mech. 14 (1965), 485-506. MR 30 \#5319.

16. M. A. Kervaire, Some nonstable homotopy groups of Lie groups, Illinois J. Math. 4 (1960), 161-169. MR $22 \# 4075$.

17. M. A. Kervaire and J. W. Milnor, Groups of homotopy spheres. I, Ann. of Math. (2) 77 (1963), 504-537. MR 26 \#5584.

18. A. Kosinski, On the inertia group of $\pi$-manifolds, Amer. J. Math. 89 (1967), 227248. MR 35 \#4936.

19. J. Levine, Self-equivalences of $S^{n} \times S^{k}$, Trans. Amer. Math. Soc. 143 (1969), 523-543. MR 40 \#2098.

20. G. R. Livesay and C. B. Thomas, Free $Z_{2}$ and $Z_{3}$ actions on homotopy spheres, Topology 7 (1968), 11-14. MR 36 \#3343.

21. S. López de Medrano, Involutions on manifolds, Ergebnisse der Mathematik und ihrer Grenzgebiete, Band 59, Springer-Verlag, New York, 1971. MR 45 \#7747.

22. J. W. Milnor, Differentiable structures on spheres, Amer. J. Math. 81 (1959), 962972. MR $22 \# 990$.

23. R. E. Mosher and M. C. Tangora, Cohomology operations and application in homotopy theory, Harper \& Row, New York and London, 1968. MR 37 \#2223.

24. P. Olum, Mappings of manifolds and the notion of degree, Ann. of Math. (2) 58 (1953), 458-480. MR 15, 338.

25. Cocycle formulas for homotopy classification; maps into projective and lens space, Trans. Amer. Math. Soc. 103 (1962), 30-44. MR 25 \#576. 
26. P. Orlik, On the Arf invariant of an involution, Canad. J. Math. 22 (1970), 519524. MR 41 \#7718.

27. D. Puppe, Homotopiemengen und ihre induzierten Abbildungen. I, Math. Z. 69 (1958), 299-344. MR 20 \#6698.

28. M. Rothenberg and J. Sondow, Non-linear smooth representatives of compact lie groups (preprint).

29. H. Sato, Diffeomeophism groups of $S^{p} \times S^{q}$ and exotic spheres, Quart. J. Math. Oxford Ser. (2) 20 (1969), 255-276. MR 40 \#6584.

30. N. E. Steenrod, The topology of fibre bundles, Princeton Math. Ser., vol. 14, Princeton Univ. Press, Princeton, N. J., 1951. MR 12, 522.

31. E. Stone, Ph. D. Thesis, Cornell University, 1972 (to appear).

32. R. Thom, Quelques propriétés globales des variétés différentiables, Comment. Math. Helv. 28 (1954), 17-86. MR 15, 890.

33. C. T. C. Wall, Free piecewise linear involutions on spheres, Bull. Amer. Math. Soc. 74 (1968), 554-558. MR 36 \#5955.

34. - Surgery on compact manifolds, Academic Press, New York, 1972.

DEPARTMENT OF MATHEMATICS, CORNELL UNIVERSITY, ITHACA, NEW YORK 14853

Current address: Department of Mathematics, University of Kansas, Lawrence, Kansas 66045 